\title{
Article \\ Plasma Carotenoids and Premenstrual Symptoms in a Multi-Ethnic Population of Young Women
}

\author{
Sophia Kerzner ${ }^{\dagger}$, Tara Zeitoun ${ }^{\dagger}(\mathbb{D}$, Alicia Jarosz, Bibiana Garcia-Bailo and Ahmed El-Sohemy * \\ Department of Nutritional Sciences, Temerty Faculty of Medicine, University of Toronto, \\ Toronto, ON M5S 1A8, Canada; sophia.kerzner@medportal.ca (S.K.); tara.zeitoun@mail.utoronto.ca (T.Z.); \\ alicia.jarosz@mail.utoronto.ca (A.J.); bibiana.garcia.bailo@mail.utoronto.ca (B.G.-B.) \\ * Correspondence: a.el.sohemy@utoronto.ca; Tel.: +1-416-946-5776 \\ + These authors contributed equally to this work.
}

check for updates

Citation: Kerzner, S.; Zeitoun, T.;

Jarosz, A.; Garcia-Bailo, B.;

El-Sohemy, A. Plasma Carotenoids and Premenstrual Symptoms in a Multi-Ethnic Population of Young Women. Nutrients 2021, 13, 3870.

https://doi.org/10.3390/nu13113870

Academic Editor: Dariusz Nowak

Received: 5 October 2021

Accepted: 27 October 2021

Published: 29 October 2021

Publisher's Note: MDPI stays neutral with regard to jurisdictional claims in published maps and institutional affiliations.

Copyright: (c) 2021 by the authors. Licensee MDPI, Basel, Switzerland. This article is an open access article distributed under the terms and conditions of the Creative Commons Attribution (CC BY) license (https:// creativecommons.org/licenses/by/ $4.0 /)$.

\begin{abstract}
Premenstrual symptoms are experienced by most women of reproductive age, but effective therapies are limited. Carotenoids may have an attenuating effect on premenstrual symptoms; however, studies to date are equivocal. The objective of the present study was to examine the association between plasma concentrations of seven carotenoids and premenstrual symptom severity in 553 women from the Toronto Nutrigenomics and Health study. Participants provided information on fifteen common premenstrual symptoms and severities. Each participant completed a General Health and Lifestyle Questionnaire and provided a fasting blood sample from which plasma carotenoid concentrations were measured. Multinomial logistic regressions were used to determine associations between plasma carotenoid concentrations and premenstrual symptom severity. Beta-cryptoxanthin was associated with moderate/severe increased appetite for women in the highest compared to the lowest tertile (OR: 2.33; 95\% CI: 1.39, 3.89). This association remained significant after adjusting for multiple comparisons. There were no observed associations between other plasma carotenoids and any premenstrual symptoms. In summary, higher concentrations of beta-cryptoxanthin were associated with an increased appetite as a premenstrual symptom, but no associations were observed for any other carotenoid and for any other symptom.
\end{abstract}

Keywords: vitamin A; retinol; carotenoids; PMS; premenstrual symptoms

\section{Introduction}

Premenstrual Syndrome (PMS) is characterized by a cluster of symptoms that present themselves during the late luteal phase of the menstrual cycle and cease a few days into menses [1]. For women with PMS, these symptoms must be recurring and followed by a symptom-free phase until the end of ovulation. Premenstrual symptoms can include physical, behavioural and psychological effects on women, such as headaches, bloating, fatigue, mood swings, acne, breast pain, anxiety and depression [2-4]. Up to 99\% of women of reproductive age experience premenstrual symptoms [1,3]. However, considerable variation exists in the type and severity of symptoms that women experience. Some of this variation may be due, in part, to factors such as differences in lifestyle, age, genetics, diet and nutritional status [5-9].

Carotenoids are known to contribute to antioxidant defence $[10,11]$, the process by which the body can protect against the effects of reactive oxygen species. Premenstrual symptoms have been associated with an inflammatory process, as evidenced by the positive correlation between their severity and the presence of interleukins and interferongamma [12]. Antioxidants are known to have a protective role in reducing inflammation [13], so it is possible that they might protect against certain premenstrual symptoms.

Vitamin A is an essential fat-soluble vitamin that plays an important role in reproduction through its effects on tissue development, cell differentiation and proliferation [14]. This micronutrient also plays a crucial role in the metabolism of macromolecules, proper 
vision, brain and immune function [15]. Vitamin A is supplied to the body as either preformed vitamin A or provitamin A, which can be converted into its biologically active form once in the body. Retinol, or preformed vitamin A, supplies approximately $60 \%$ of an individual's average daily vitamin A intake through animal products and by-products such as liver, kidney, eggs, fatty fish and dairy [16,17]. The other $40 \%$ is supplied by provitamin $\mathrm{A}$, which includes carotenoids such as beta-carotene, alpha-carotene, and betacryptoxanthin [17]. Common dietary sources of provitamin A include dark leafy greens and fruits and vegetables that are red or orange in colour [16,17]. Circulating carotenoids serve as robust biomarkers of fruit and vegetable intake [18].

The potential effects of carotenoids in the amelioration of premenstrual symptoms could involve their antioxidant capacity $[11,13]$. However, the evidence is limited, and previous studies have yielded inconsistent results [19-22]. The objective of the present study was to examine the association between plasma concentrations of seven individual carotenoids and premenstrual symptom severity in a multi-ethnic population of young women.

\section{Materials and Methods}

The study participants $(n=553)$ consisted of women of reproductive age from the Toronto Nutrigenomics and Health (TNH) Study. The TNH study is a cross-sectional examination of 1640 young adults aged 20-29 years, living in Toronto and recruited between 2004 and 2010. Women who were breastfeeding or pregnant were excluded from the study. Details of the study have been described previously [23]. Participants completed a General Health and Lifestyle Questionnaire (GHLQ), which included lifestyle, demographic and medical history questions, hormonal contraceptive use and information on physical activity levels (PAL). Fifteen premenstrual symptoms and their respective severities were also selfreported in the GHLQ. The participants recorded their dietary intake via a 196-item Torontomodified Harvard food frequency questionnaire, had anthropometric measurements taken, and provided an overnight fasting blood sample. The TNH study was approved by the University of Toronto Research Ethics Board.

Exclusion criteria for the present study included male sex $(n=523)$, those with a reported diagnosis of endometriosis, amenorrhea or polycystic ovarian syndrome $(n=14)$, smokers $(n=53)$, the use of hormonal contraceptives $(n=308)$ and the use of anxiolytics or anti-depressants $(n=25)$, as these medications may affect the severity and/or prevalence of premenstrual symptoms. Individuals with missing GHLQ or plasma carotenoid data $(n=164)$ were also excluded. After all exclusions, the total sample size was 553 women. Participants in this study were categorized into four ethnocultural groups based on selfidentification: Caucasian $(n=197)$, East Asian $(n=254)$, South Asian $(n=62)$ or Other $(n=40)$. Caucasians included participants who reported being of European, Middle Eastern, or Hispanic origin. East Asians consisted of individuals of Chinese, Japanese, Korean, Vietnamese, Filipino, Thai or Cambodian origin. South Asians self-reported as Bangladeshi, Indian, Pakistani or Sri Lankan. Participants in the "Other" category included Indigenous Canadians, Afro-Caribbeans and those who were self-reported as belonging to two or more ethnocultural groups.

Participants' anthropometric variables were measured, including their height, weight, waist circumference and blood pressure, as described previously [3]. Subjects' body mass index (BMI) was calculated in $\mathrm{kg} / \mathrm{m}^{2}$. Subjects were also asked to self-report on their PAL in the GHLQ, including time spent sleeping and engaging in light to vigorous physical activity. Physical activity values were converted to metabolic equivalent (MET) hours per week [3].

Each participant provided a blood sample following a minimum 12-h overnight fast. Seven plasma carotenoids were measured: beta-carotene, alpha-carotene, retinol, lutein, zeaxanthin, beta-cryptoxanthin and trans-lycopene. Plasma carotenoid concentrations were measured by high-performance liquid chromatography-tandem mass spectrometry as previously described [24].

Premenstrual symptom prevalence and severity were self-reported by each female participant in the GHLQ. Women indicated the degree to which they experienced each 
symptom within 5 days prior to the onset of their period and ending by the 4th day of their period. The questionnaire classified severity as: none, mild, moderate and severe. The questionnaire included the following symptoms: acne, skin blemish; bloating, swelling, breast tenderness; cramping; mood swings, crying easily, irritability, angry outbursts; increased appetite, food cravings; fatigue; headaches; anxiety, tension, nervousness; clumsiness; confusion, difficulty concentrating, forgetfulness; sexual desire/activity change; insomnia; nausea; depression; desire to be alone and other, based on symptoms commonly reported in the literature and previously validated questionnaires [3]. Subjects that selected "other" were asked to specify the symptom; however, due to an insufficient number of responses in this category, these were not included in the analyses.

All statistical analyses were performed using RStudio (version 1.3.1). The $\alpha$ error was set at 0.05 and all reported $p$-values are two-sided. The distribution of continuous variables was assessed before analysis to evaluate normality; body mass index (BMI) was non-normally distributed and was, therefore, log-transformed for analysis. Premenstrual symptom severity was categorized as none, mild or moderate/severe. The moderate and severe symptom groups were merged into one category for analysis because of the low prevalence of reporting severe symptoms. Plasma carotenoids were categorized into tertiles, with the lowest tertile set as the reference.

Multinomial logistic regressions were used to determine associations between plasma carotenoid concentrations and the severity of 15 common premenstrual symptoms for betacarotene, alpha-carotene, retinol, lutein, zeaxanthin, beta-cryptoxanthin and trans-lycopene. $p$-values were calculated for both unadjusted and adjusted models. The adjusted multinomial logistic regressions included the following covariates: age, ethnicity, PAL, medication use, log-transformed BMI and plasma triglycerides. Covariates were selected based on their associations with premenstrual symptoms or plasma carotenoid concentrations in the TNH study population and previous studies. Benjamini-Yekutieli (BY) adjustments for multiple comparisons were applied $(\alpha=0.05: p<0.0083)$ [25]. Odds ratios and $95 \%$ confidence intervals (CI) were reported for all associations.

\section{Results}

The prevalence of reporting premenstrual symptoms among female TNH Study participants is shown in Table 1 . Nearly every woman (99\%) in the study population reported experiencing at least one premenstrual symptom, underscoring the high prevalence of this condition among young women during their ovulatory years. Cramps were the most reported premenstrual symptom (76\%), followed by mood swings $(73 \%)$, bloating $(71 \%)$ and increased appetite $(61 \%)$. Among these top four reported symptoms, approximately $30 \%$ to $40 \%$ of women reported experiencing them at a moderate or severe level. Insomnia was the least experienced symptom $(12 \%)$, with only $3 \%$ of women reporting its severity as moderate or higher.

Table 1. Prevalence of reporting of premenstrual symptoms among 553 women.

\begin{tabular}{|c|c|c|c|c|}
\hline \multirow{2}{*}{ Premenstrual Symptoms } & \multicolumn{3}{|c|}{ Prevalence by Degree of Reported Severity $(n(\%))$} & \multirow{2}{*}{ Total Presence of Symptoms } \\
\hline & None & Mild & Moderate/Severe & \\
\hline Any symptom & & & & 549 (99) \\
\hline Cramps & $130(23)$ & $187(34)$ & $236(43)$ & $423(76)$ \\
\hline Mood swings/crying easily/irritability/angry outbursts & $152(27)$ & $208(38)$ & $193(35)$ & $401(73)$ \\
\hline Bloating/swelling/breast tenderness & $158(29)$ & $213(38)$ & $182(33)$ & $395(71)$ \\
\hline Increased appetite/food Cravings & $212(38)$ & $160(29)$ & $181(33)$ & $341(61)$ \\
\hline Acne/skin blemish & $226(41)$ & $214(39)$ & $113(20)$ & $327(59)$ \\
\hline Fatigue & $247(45)$ & $176(32)$ & $130(23)$ & $306(55)$ \\
\hline Sexual desire/activity change & $283(51)$ & $177(32)$ & $93(17)$ & $270(49)$ \\
\hline Desire to be alone & $351(63)$ & $136(25)$ & $62(12)$ & $202(37)$ \\
\hline Anxiety/tension/nervousness & $352(64)$ & $129(23)$ & $77(13)$ & $201(36)$ \\
\hline Depression & $398(72)$ & $98(18)$ & $57(10)$ & $155(28)$ \\
\hline Headache & $412(75)$ & $90(16)$ & $51(9)$ & $141(24)$ \\
\hline Confusion/difficulty concentrating/forgetfulness & $423(76)$ & $87(16)$ & $43(8)$ & $130(23)$ \\
\hline Nausea & $464(84)$ & $68(12)$ & $21(4)$ & $89(16)$ \\
\hline Clumsiness & $470(85)$ & $59(10)$ & $25(5)$ & $83(15)$ \\
\hline Insomnia & $485(88)$ & $52(9)$ & $16(3)$ & $68(12)$ \\
\hline
\end{tabular}


Multinomial logistic regressions were conducted to examine the associations between plasma carotenoid concentrations and premenstrual symptom severity. Tables $2-8$ show the results for beta-cryptoxanthin (Table 2), beta-carotene (Table 3), alpha-carotene (Table 4), retinol (Table 5), lutein (Table 6), zeaxanthin (Table 7) and trans-lycopene (Table 8). We found several statistically significant associations between specific plasma carotenoids and the severity of premenstrual symptoms after adjusting for age, log-transformed BMI, ethnicity, PAL, use of analgesics and plasma triglyceride levels. However, only a few remained significant after adjusting for multiple comparisons. Women in the highest tertile of plasma beta-cryptoxanthin levels were more likely to experience moderate-severe appetite changes (OR: 2.33; 95\% CI: 1.39, 3.89) compared to those in the lowest tertile of beta-cryptoxanthin levels (Table 2). This observation was made both in the unadjusted and adjusted models, (OR: 2.10; 95\% CI: 1.29, 3.44) and (OR: 2.33; 95\% CI: 1.39, 3.89), respectively. Compared to those in the lowest tertile for plasma beta-carotene, those in the second tertile had a decreased risk of experiencing moderate-severe premenstrual headaches in both the unadjusted and adjusted models, (OR: 0.33; 95\% CI: 0.15, 0.73) and (OR: 0.29; 95\% CI: 0.13, 0.68), respectively (Table 3). Women in the second tertile of plasma alpha-carotene were less likely to experience mild premenstrual nausea (OR: 0.35; 95\% CI: $0.17,0.73)$ compared to those in the lowest tertile, although this association was attenuated and no longer significant after adjusting for covariates (Table 4). Women in the second tertile with intermediate retinol plasma concentrations were more likely to experience moderate/severe premenstrual sexual desire changes compared to those in the lowest tertile (Table 5). This association was observed in both unadjusted and adjusted models (OR: 2.35; 95\% CI: 1.31, 4.23) and (OR: 2.17; 95\% CI: 1.17, 4.01), respectively. There was an inverse association between high plasma lutein concentrations and moderate/severe premenstrual cramps (OR: 0.48; 95\% CI: 0.29, 0.81); however, this association was attenuated after adjustment for covariates (Table 6). Women in the second tertile of plasma lutein levels had lower odds of experiencing mild premenstrual mood swings in comparison to those with low plasma lutein levels (OR: $0.48 ; 95 \%$ CI: $0.28,0.83$ ). This association was observed after adjusting for potential covariates only. There were no observed associations between plasma trans-lycopene or plasma zeaxanthin with any premenstrual symptom severities (Tables 7 and 8). 
Table 2. Unadjusted and adjusted Odds Ratios and 95\% CIs for the association between tertiles of plasma beta-cryptoxanthin and premenstrual symptoms.

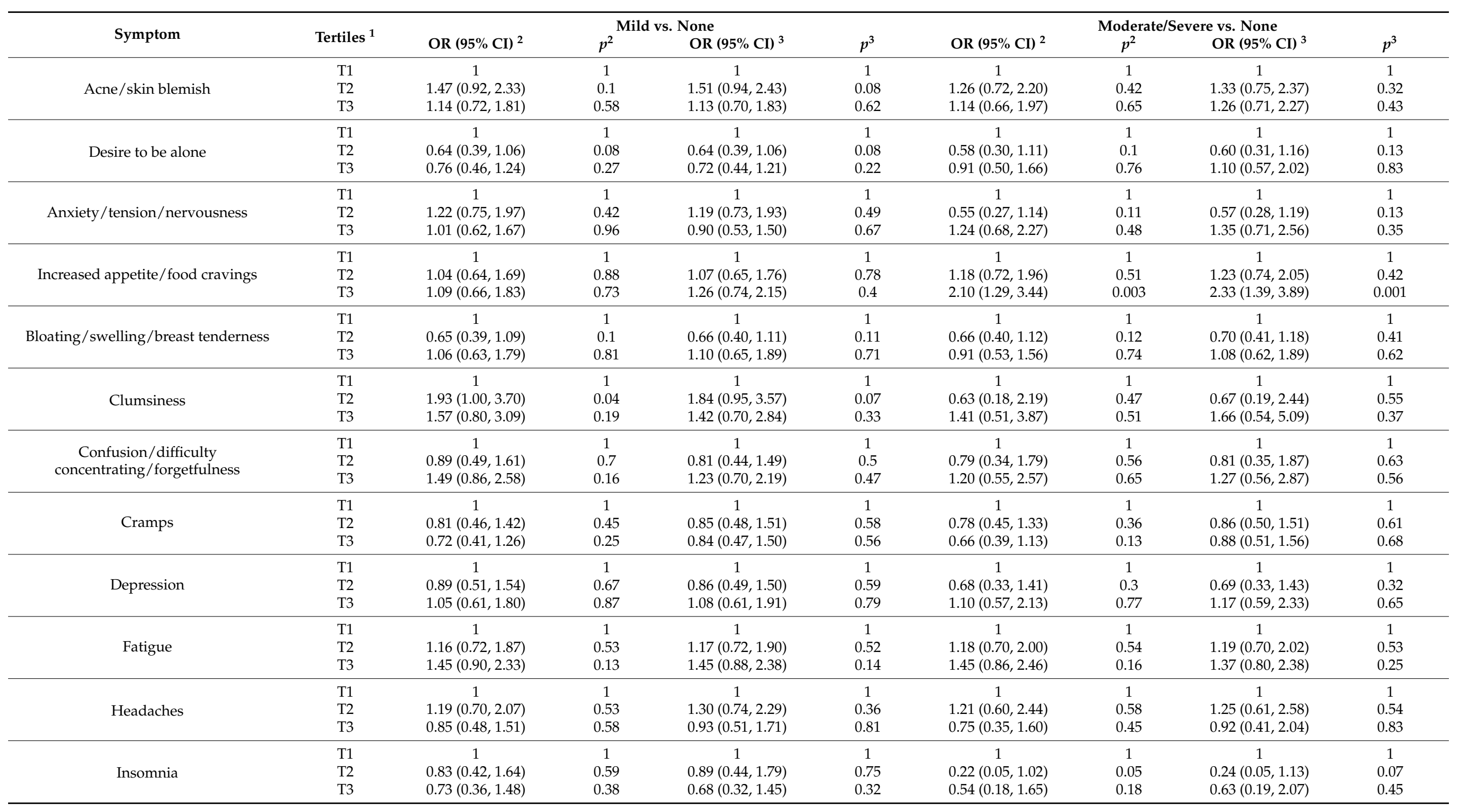


Table 2. Cont.

\begin{tabular}{|c|c|c|c|c|c|c|c|c|c|}
\hline \multirow{2}{*}{ Symptom } & \multirow{2}{*}{ Tertiles $^{1}$} & \multicolumn{4}{|c|}{ Mild vs. None } & \multicolumn{4}{|c|}{ Moderate/Severe vs. None } \\
\hline & & OR $(95 \% \mathrm{CI})^{2}$ & $p^{2}$ & OR $(95 \% \mathrm{CI})^{3}$ & $p^{3}$ & OR $(95 \% \mathrm{CI})^{2}$ & $p^{2}$ & OR $(95 \% \mathrm{CI})^{3}$ & $p^{3}$ \\
\hline \multirow{3}{*}{$\begin{array}{l}\text { Mood swings/crying } \\
\text { easily/irritability/angry outbursts }\end{array}$} & $\mathrm{T} 1$ & 1 & 1 & 1 & 1 & 1 & 1 & 1 & 1 \\
\hline & $\mathrm{T} 2$ & $1.19(0.71,1.99)$ & 0.5 & $1.24(0.73,2.09)$ & 0.42 & $1.06(0.62,1.79)$ & 0.84 & $1.11(0.65,1.89)$ & 0.7 \\
\hline & T3 & $0.90(0.54,1.52)$ & 0.71 & $0.82(0.48,1.41)$ & 0.48 & $1.14(0.68,1.90)$ & 0.62 & $1.22(0.71,2.09)$ & 0.47 \\
\hline \multirow{3}{*}{ Nausea } & T1 & 1 & 1 & 1 & 1 & 1 & 1 & 1 & 1 \\
\hline & $\mathrm{T} 2$ & $1.87(0.97,3.62)$ & 0.06 & $1.96(0.99,3.84)$ & 0.05 & $0.98(0.37,2.63)$ & 0.98 & $1.25(0.45,3.47)$ & 0.67 \\
\hline & $\mathrm{T} 3$ & $0.95(0.46,2.00)$ & 0.9 & $1.17(0.54,2.55)$ & 0.68 & $0.91(0.34,2.41)$ & 0.84 & $1.58(0.53,4.67)$ & 0.41 \\
\hline \multirow{3}{*}{ Sexual desire/activity change } & T1 & 1 & 1 & 1 & 1 & 1 & 1 & 1 & 1 \\
\hline & $\mathrm{T} 2$ & $0.96(0.45,1.15)$ & 0.87 & $0.99(0.62,1.58)$ & 0.96 & $0.58(0.33,1.03)$ & 0.06 & $0.61(0.34,1.11)$ & 0.11 \\
\hline & $\mathrm{T} 3$ & $1.05(0.47,1.21)$ & 0.66 & $1.08(0.67,1.76)$ & 0.74 & $0.65(0.37,1.15)$ & 0.14 & $0.94(0.51,1.74)$ & 0.86 \\
\hline
\end{tabular}

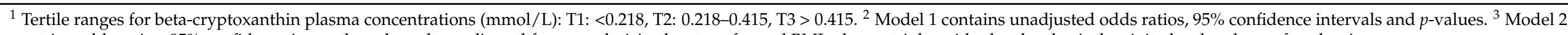
contains odds ratios, $95 \%$ confidence intervals and $p$-values adjusted for age, ethnicity, log-transformed BMI, plasma triglycerides levels, physical activity level and use of analgesics.

Table 3. Unadjusted and adjusted Odds Ratios and 95\% CIs for the association between tertiles of plasma beta-carotene and premenstrual symptoms.

\begin{tabular}{|c|c|c|c|c|c|c|c|c|c|}
\hline \multirow{2}{*}{ Symptom } & \multirow[b]{2}{*}{ Tertiles $^{1}$} & \multicolumn{4}{|c|}{ Mild vs. None } & \multicolumn{4}{|c|}{ Moderate/Severe vs. None } \\
\hline & & OR $(95 \% \mathrm{CI})^{2}$ & $p^{2}$ & OR $(95 \% \mathrm{CI})^{3}$ & $p^{3}$ & OR $(95 \% \mathrm{CI})^{2}$ & $p^{2}$ & OR $(95 \% \mathrm{CI})^{3}$ & $p^{3}$ \\
\hline \multirow{3}{*}{ Acne/skin blemish } & $\mathrm{T} 1$ & 1 & 1 & 1 & 1 & 1 & 1 & 1 & 1 \\
\hline & T2 & $0.96(0.61,1.53)$ & 0.88 & $0.93(0.57,1.50)$ & 0.76 & $1.26(0.72,2.18)$ & 0.42 & $1.29(0.72,2.31)$ & 0.38 \\
\hline & $\mathrm{T} 3$ & $1.25(0.80,1.98)$ & 0.32 & $1.16(0.72,1.89)$ & 0.53 & $1.29(0.74,2.28)$ & 0.37 & $1.37(0.75,2.51)$ & 0.3 \\
\hline \multirow{3}{*}{ Desire to be alone } & $\mathrm{T} 1$ & 1 & 1 & 1 & 1 & 1 & 1 & 1 & 1 \\
\hline & $\mathrm{T} 2$ & $1.19(0.73,1.93)$ & 0.47 & $1.17(0.71,1.94)$ & 0.52 & $0.84(0.46,1.55)$ & 0.58 & $1.00(0.53,1.91)$ & 0.99 \\
\hline & $\mathrm{T} 3$ & $0.68(0.40,1.13)$ & 0.14 & $0.64(0.37,1.09)$ & 0.1 & $0.66(0.35,1.22)$ & 0.18 & $0.87(0.45,1.69)$ & 0.68 \\
\hline \multirow{3}{*}{ Anxiety/tension/nervousness } & T1 & 1 & 1 & 1 & 1 & 1 & 1 & 1 & 1 \\
\hline & $\mathrm{T} 2$ & $0.98(0.61,1.58)$ & 0.93 & $0.91(0.55,1.50)$ & 0.71 & $0.66(0.33,1.33)$ & 0.25 & $0.63(0.31,1.29)$ & 0.21 \\
\hline & $\mathrm{T} 3$ & $0.86(053,1.41)$ & 0.56 & $0.78(0.47,1.34)$ & 0.39 & $1.16(0.63,2.15)$ & 0.63 & $1.12(0.58,2.14)$ & 0.74 \\
\hline \multirow{3}{*}{ Increased appetite/food cravings } & $\mathrm{T} 1$ & 1 & 1 & 1 & 1 & 1 & 1 & 1 & 1 \\
\hline & $\mathrm{T} 2$ & $1.09(0.66,1.80)$ & 0.72 & $1.26(0.75,2.12)$ & 0.37 & $1.28(0.78,2.09)$ & 0.32 & $1.39(0.74,2.05)$ & 0.2 \\
\hline & $\mathrm{T} 3$ & $1.19(0.72,1.98)$ & 0.48 & $1.38(0.82,2.36)$ & 0.23 & $1.54(0.94,2.51)$ & 0.08 & $1.70(1.01,2.84)$ & 0.04 \\
\hline \multirow{3}{*}{ Bloating/swelling/breast tenderness } & $\mathrm{T} 1$ & 1 & 1 & 1 & 1 & 1 & 1 & 1 & 1 \\
\hline & $\mathrm{T} 2$ & $0.82(0.50,1.36)$ & 0.45 & $0.81(0.48,1.37)$ & 0.44 & $0.77(0.46,1.31)$ & 0.34 & $0.85(0.50,1.49)$ & 0.6 \\
\hline & T3 & $0.82(0.49,1.36)$ & 0.44 & $0.99(0.47,1.38)$ & 0.44 & $0.87(0.52,1.48)$ & 0.62 & $0.99(0.57,1.74)$ & 0.99 \\
\hline \multirow{3}{*}{ Clumsiness } & $\mathrm{T} 1$ & 1 & 1 & 1 & 1 & 1 & 1 & 1 & 1 \\
\hline & $\mathrm{T} 2$ & $0.86(0.46,1.59)$ & 0.62 & $0.84(0.44,1.60)$ & 0.6 & $0.69(0.22,2.24)$ & 0.54 & $0.84(0.24,2.91)$ & 0.78 \\
\hline & T3 & $0.83(0.45,1.55)$ & 0.57 & $0.79(0.40,1.52)$ & 0.48 & $1.28(0.46,3.52)$ & 0.64 & $1.70(0.55,5.24)$ & 0.35 \\
\hline
\end{tabular}


Table 3. Cont.

\begin{tabular}{|c|c|c|c|c|c|c|c|c|c|}
\hline \multirow{2}{*}{ Symptom } & \multirow{2}{*}{ Tertiles $^{1}$} & \multicolumn{4}{|c|}{ Mild vs. None } & \multicolumn{4}{|c|}{ Moderate/Severe vs. None } \\
\hline & & OR $(95 \% \mathrm{CI})^{2}$ & $p^{2}$ & OR $(95 \% \text { CI })^{3}$ & $p^{3}$ & OR $(95 \% \text { CI })^{2}$ & $p^{2}$ & OR $(95 \% \mathrm{CI})^{3}$ & $p^{3}$ \\
\hline \multirow{3}{*}{$\begin{array}{l}\text { Confusion/difficulty } \\
\text { concentrating/forgetfulness }\end{array}$} & $\mathrm{T} 1$ & 1 & 1 & 1 & 1 & 1 & 1 & 1 & 1 \\
\hline & $\mathrm{T} 2$ & $1.16(0.65,2.05)$ & 0.7 & $1.14(0.62,2.08)$ & 0.67 & $0.43(0.18,1.03)$ & 0.05 & $0.42(0.17,1.02)$ & 0.05 \\
\hline & $\mathrm{T} 3$ & $1.19(0.67,2.11)$ & 0.55 & $1.09(0.59,2.03)$ & 0.76 & $0.97(0.48,1.96)$ & 0.94 & $0.96(0.50,2.03)$ & 0.91 \\
\hline \multirow{3}{*}{ Cramps } & T1 & 1 & 1 & 1 & 1 & 1 & 1 & 1 & 1 \\
\hline & $\mathrm{T} 2$ & $1.09(0.62,1.91)$ & 0.76 & $1.29(0.72,2.32)$ & 0.39 & $0.63(0.45,1.33)$ & 0.08 & $0.39(0.44,1.38)$ & 0.39 \\
\hline & T3 & $0.88(0.50,1.54)$ & 0.66 & $1.00(0.55,1.81)$ & 0.99 & $0.58(0.34,0.98)$ & 0.04 & $0.20(0.39,1.22)$ & 0.2 \\
\hline \multirow{3}{*}{ Depression } & $\mathrm{T} 1$ & 1 & 1 & 1 & 1 & 1 & 1 & 1 & 1 \\
\hline & $\mathrm{T} 2$ & $1.12(0.67,1.88)$ & 0.66 & $1.13(0.65,1.94)$ & 0.66 & $0.81(0.40,1.63)$ & 0.55 & $0.81(0.39,1.67)$ & 0.57 \\
\hline & T3 & $0.64(0.36,1.14)$ & 0.13 & $0.66(0.36,1.21)$ & 0.18 & $0.98(0.51,1.90)$ & 0.96 & $1.00(0.50,2.03)$ & 0.98 \\
\hline \multirow{3}{*}{ Fatigue } & T1 & 1 & 1 & 1 & 1 & 1 & 1 & 1 & 1 \\
\hline & $\mathrm{T} 2$ & $1.09(0.68,1.76)$ & 0.71 & $1.07(0.66,1.76)$ & 0.77 & $1.37(0.82,2.31)$ & 0.23 & $1.39(0.81,2.39)$ & 0.24 \\
\hline & $\mathrm{T} 3$ & $1.16(0.72,1.85)$ & 0.54 & $1.15(0.70,1.89)$ & 0.57 & $1.21(0.72,2.06)$ & 0.47 & $1.18(0.67,2.07)$ & 0.56 \\
\hline \multirow{3}{*}{ Headaches } & T1 & 1 & 1 & 1 & 1 & 1 & 1 & 1 & 1 \\
\hline & $\mathrm{T} 2$ & $1.27(0.73,2.20)$ & 0.4 & $1.58(0.87,2.85)$ & 0.13 & $0.33(0.15,0.73)$ & 0.006 & $0.29(0.13,0.68)$ & 0.004 \\
\hline & T3 & $0.94(0.52,1.68)$ & 0.82 & $1.22(0.65,2.30)$ & 0.54 & $0.57(0.29,1.12)$ & 0.1 & $0.58(0.28,1.21)$ & 0.15 \\
\hline \multirow[b]{2}{*}{ Insomnia } & T1 & 1 & 1 & 1 & 1 & 1 & 1 & 1 & 1 \\
\hline & T2 & $1.15(0.58,2.29)$ & 0.68 & $1.23(0.62,2.61)$ & 0.51 & $0.22(0.04,1.03)$ & 0.053 & $0.22(0.04,1.04)$ & 0.056 \\
\hline \multirow{3}{*}{$\begin{array}{c}\text { Mood swings/crying } \\
\text { easily/irritability/angry outbursts }\end{array}$} & $\mathrm{T} 1$ & 1 & 1 & 1 & 1 & 1 & 1 & 1 & 1 \\
\hline & $\mathrm{T} 2$ & $1.06(0.63,1.77)$ & 0.83 & $0.94(0.55,1.60)$ & 0.81 & $1.18(0.69,2.00)$ & 0.55 & $1.21(0.70,2.10)$ & 0.5 \\
\hline & T3 & $0.75(0.45,1.24)$ & 0.26 & $0.62(0.36,1.07)$ & 0.08 & $0.97(0.58,1.62)$ & 0.9 & $1.04(0.60,1.80)$ & 0.9 \\
\hline \multirow{3}{*}{ Nausea } & $\mathrm{T} 1$ & 1 & 1 & 1 & 1 & 1 & 1 & 1 & 1 \\
\hline & $\mathrm{T} 2$ & $0.76(0.39,1.49)$ & 0.43 & $0.81(0.40,1.63)$ & 0.55 & $1.23(0.47,3.21)$ & 0.66 & $1.53(0.55,4.25)$ & 0.41 \\
\hline & $\mathrm{T} 3$ & $0.85(0.44,1.63)$ & 0.62 & $0.90(0.45,1.81)$ & 0.77 & $0.86(0.30,2.42)$ & 0.77 & $1.14(0.37,3.50)$ & 0.82 \\
\hline \multirow{3}{*}{ Sexual desire/activity change } & $\mathrm{T} 1$ & 1 & 1 & 1 & 1 & 1 & 1 & 1 & 1 \\
\hline & $\mathrm{T} 2$ & $0.81(0.51,1.28)$ & 0.36 & $0.80(0.49,1.29)$ & 0.34 & $0.68(0.39,1.21)$ & 0.19 & $0.80(0.44,1.46)$ & 0.46 \\
\hline & T3 & $0.81(0.51,1.29)$ & 0.38 & $0.83(0.51,1.34)$ & 0.44 & $0.63(0.35,1.13)$ & 0.12 & $0.80(0.43,1.50)$ & 0.5 \\
\hline
\end{tabular}

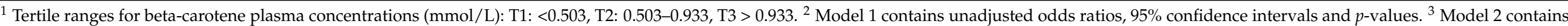

odds ratios, $95 \%$ confidence intervals and $p$-values adjusted for age, ethnicity, log-transformed BMI, plasma triglycerides levels, physical activity level and use of analgesics. 
Table 4. Unadjusted and adjusted Odds Ratios and 95\% CIs for the association between tertiles of plasma alpha-carotene and premenstrual symptoms.

\begin{tabular}{|c|c|c|c|c|c|c|c|c|c|}
\hline \multirow{2}{*}{ Symptom } & \multirow{2}{*}{ Tertiles $^{1}$} & \multicolumn{4}{|c|}{ Mild vs. None } & \multicolumn{4}{|c|}{ Moderate/Severe vs. None } \\
\hline & & OR $(95 \% \mathrm{CI})^{2}$ & $p^{2}$ & OR $(95 \% \mathrm{CI})^{3}$ & $p^{3}$ & OR $(95 \% \mathrm{CI})^{2}$ & $p^{2}$ & OR $(95 \% \mathrm{CI})^{3}$ & $p^{3}$ \\
\hline \multirow{3}{*}{ Acne/skin blemish } & $\mathrm{T} 1$ & 1 & 1 & 1 & 1 & 1 & 1 & 1 & 1 \\
\hline & $\mathrm{T} 2$ & $1.33(0.84,2.10)$ & 0.22 & $1.35(0.85,2.16)$ & 0.2 & $1.04(0.60,1.82)$ & 0.89 & $1.11(0.63,1.97)$ & 0.71 \\
\hline & $\mathrm{T} 3$ & $1.00(0.63,1.58)$ & 0.99 & $0.89(0.56,1.43)$ & 0.64 & $1.00(0.58,1.73)$ & 0.99 & $0.95(0.54,1.66)$ & 0.85 \\
\hline \multirow{3}{*}{ Desire to be alone } & $\mathrm{T} 1$ & 1 & 1 & 1 & 1 & 1 & 1 & 1 & 1 \\
\hline & $\mathrm{T} 2$ & $1.18(0.73,1.92)$ & 0.49 & $1.16(0.71,1.90)$ & 0.55 & $0.53(0.29,0.99)$ & 0.047 & $0.54(0.29,1.02)$ & 0.058 \\
\hline & T3 & $0.72(0.43,1.21)$ & 0.22 & $0.73(0.43,1.23)$ & 0.23 & $0.48(0.26,0.90)$ & 0.02 & $0.55(0.29,1.04)$ & 0.06 \\
\hline \multirow{3}{*}{ Anxiety/tension/nervousness } & $\mathrm{T} 1$ & 1 & 1 & 1 & 1 & 1 & 1 & 1 & 1 \\
\hline & $\mathrm{T} 2$ & $1.11(0.70,1.78)$ & 0.06 & $1.09(0.68,1.75)$ & 0.72 & $0.61(0.32,1.19)$ & 0.15 & $0.60(0.31,1.18)$ & 0.14 \\
\hline & $\mathrm{T} 3$ & $0.60(0.36,1.01)$ & 0.054 & $0.58(0.35,0.98)$ & 0.043 & $0.72(0.39,1.33)$ & 0.29 & $0.68(0.36,1.29)$ & 0.24 \\
\hline \multirow{3}{*}{ Increased appetite/food cravings } & $\mathrm{T} 1$ & 1 & 1 & 1 & 1 & 1 & 1 & 1 & 1 \\
\hline & $\mathrm{T} 2$ & $1.12(0.69,1.85)$ & 0.64 & $1.22(0.74,2.01)$ & 0.44 & $1.16(0.71,1.89)$ & 0.54 & $1.21(0.74,1.98)$ & 0.45 \\
\hline & $\mathrm{T} 3$ & $1.07(0.64,1.77)$ & 0.8 & $1.10(0.66,1.85)$ & 0.71 & $1.34(0.82,2.17)$ & 0.24 & $1.35(0.82,2.22)$ & 0.23 \\
\hline \multirow{3}{*}{ Bloating/swelling/breast tenderness } & $\mathrm{T} 1$ & 1 & 1 & 1 & 1 & 1 & 1 & 1 & 1 \\
\hline & $\mathrm{T} 2$ & $0.87(0.52,1.44)$ & 0.58 & $0.86(0.52,1.44)$ & 0.57 & $0.74(0.44,1.25)$ & 0.26 & $0.79(0.46,1.35)$ & 0.39 \\
\hline & $\mathrm{T} 3$ & $0.79(0.48,1.32)$ & 0.37 & $0.78(0.47,1.31)$ & 0.35 & $0.71(0.42,1.21)$ & 0.21 & $0.73(0.43,1.25)$ & 0.25 \\
\hline \multirow[b]{2}{*}{ Clumsiness } & $\mathrm{T} 1$ & 1 & 1 & 1 & 1 & 1 & 1 & 1 & 1 \\
\hline & $\mathrm{T} 2$ & $1.25(0.67,2.31)$ & 0.48 & $1.21(0.65,2.26)$ & 0.56 & $0.56(1.83,1.71)$ & 0.31 & $0.62(0.19,1.94)$ & 0.41 \\
\hline \multirow{3}{*}{$\begin{array}{l}\text { Confusion/difficulty } \\
\text { concentrating/forgetfulness }\end{array}$} & $\mathrm{T} 1$ & 1 & 1 & 1 & 1 & 1 & 1 & 1 & 1 \\
\hline & $\mathrm{T} 2$ & $1.49(0.85,2.62)$ & 0.16 & $1.43(0.81,2.55)$ & 0.22 & $0.69(0.32,1.49)$ & 0.34 & $0.68(0.31,1.49)$ & 0.34 \\
\hline & $\mathrm{T} 3$ & $1.05(0.58,1.90)$ & 0.88 & $1.06(0.58,1.96)$ & 0.85 & $0.73(0.34,1.54)$ & 0.4 & $0.74(0.34,1.60)$ & 0.45 \\
\hline \multirow{3}{*}{ Cramps } & T1 & 1 & 1 & 1 & 1 & 1 & 1 & 1 & 1 \\
\hline & T2 & $1.01(0.57,1.78)$ & 0.96 & $1.08(0.61,1.93)$ & 0.78 & $0.79(0.40,1.47)$ & 0.4 & $0.90(0.52,1.56)$ & 0.71 \\
\hline & $\mathrm{T} 3$ & $0.84(0.48,1.46)$ & 0.53 & $0.86(0.49,1.51)$ & 0.6 & $0.57(0.33,0.96)$ & 0.034 & $0.59(0.34,1.03)$ & 0.06 \\
\hline \multirow{3}{*}{ Depression } & $\mathrm{T} 1$ & 1 & 1 & 1 & 1 & 1 & 1 & 1 & 1 \\
\hline & $\mathrm{T} 2$ & $0.92(0.55,1.54)$ & 0.76 & $0.93(0.55,1.57)$ & 0.78 & $0.69(0.35,1.37)$ & 0.29 & $0.67(0.34,1.36)$ & 0.27 \\
\hline & $\mathrm{T} 3$ & $0.48(0.27,0.86)$ & 0.012 & $0.46(0.26,0.84)$ & 0.011 & $0.76(0.39,1.48)$ & 0.43 & $0.82(0.42,1.60)$ & 0.55 \\
\hline \multirow{3}{*}{ Fatigue } & $\mathrm{T} 1$ & 1 & 1 & 1 & 1 & 1 & 1 & 1 & 1 \\
\hline & $\mathrm{T} 2$ & $1.10(0.69,1.77)$ & 0.67 & $1.070 .67,1.73)$ & 0.76 & $1.55(0.92,2.64)$ & 0.1 & $1.52(0.89,2.59)$ & 0.13 \\
\hline & $\mathrm{T} 3$ & $0.93(0.58,1.50)$ & 0.78 & $0.95(0.59,1.54)$ & 0.85 & $1.34(0.79,2.28)$ & 0.28 & $1.40(0.81,2.41)$ & 0.23 \\
\hline \multirow{3}{*}{ Headaches } & $\mathrm{T} 1$ & 1 & 1 & 1 & 1 & 1 & 1 & 1 & 1 \\
\hline & $\mathrm{T} 2$ & $1.09(0.63,1.91)$ & 0.74 & $1.13(0.64,2.00)$ & 0.67 & $0.59(0.29,1.19)$ & 0.14 & $0.60(0.29,1.25)$ & 0.17 \\
\hline & $\mathrm{T} 3$ & $0.93(0.53,1.65)$ & 0.8 & $1.09(0.61,1.98)$ & 0.75 & $0.59(0.29,1.19)$ & 0.14 & $0.61(0.29,1.27)$ & 0.19 \\
\hline \multirow{3}{*}{ Insomnia } & $\mathrm{T} 1$ & 1 & 1 & 1 & 1 & 1 & 1 & 1 & 1 \\
\hline & T2 & $0.93(0.47,1.84)$ & 0.83 & $0.93(0.46,1.88)$ & 0.85 & $0.70(0.22,2.25)$ & 0.55 & $0.77(0.23,2.58)$ & 0.68 \\
\hline & T3 & $0.78(0.38,1.59)$ & 0.49 & $0.82(0.40,1.72)$ & 0.61 & $0.56(0.16,1.97)$ & 0.27 & $0.61(0.71,2.21)$ & 0.46 \\
\hline
\end{tabular}


Table 4. Cont.

\begin{tabular}{|c|c|c|c|c|c|c|c|c|c|}
\hline \multirow{2}{*}{ Symptom } & \multirow{2}{*}{ Tertiles $^{1}$} & \multicolumn{4}{|c|}{ Mild vs. None } & \multicolumn{4}{|c|}{ Moderate/Severe vs. None } \\
\hline & & OR $(95 \% \mathrm{CI})^{2}$ & $p^{2}$ & OR $(95 \% \text { CI })^{3}$ & $p^{3}$ & OR $(95 \% \mathrm{CI})^{2}$ & $p^{2}$ & OR $(95 \% \mathrm{CI})^{3}$ & $p^{3}$ \\
\hline \multirow{3}{*}{$\begin{array}{l}\text { Mood swings/crying } \\
\text { easily/irritability/angry outbursts }\end{array}$} & $\mathrm{T} 1$ & 1 & 1 & 1 & 1 & 1 & 1 & 1 & 1 \\
\hline & $\mathrm{T} 2$ & $1.30(0.77,2.19)$ & 0.32 & $1.25(0.74,2.12)$ & 0.41 & $1.35(0.80,2.29)$ & 0.26 & $1.37(0.80,2.34)$ & 0.25 \\
\hline & $\mathrm{T} 3$ & $0.86(0.52,1.44)$ & 0.57 & $0.79(0.47,1.33)$ & 0.37 & $0.92(0.55,1.53)$ & 0.74 & $0.94(0.56,1.59)$ & 0.82 \\
\hline \multirow{3}{*}{ Nausea } & T1 & 1 & 1 & 1 & 1 & 1 & 1 & 1 & 1 \\
\hline & $\mathrm{T} 2$ & $0.35(0.17,0.73)$ & 0.005 & $0.38(0.18,0.80)$ & 0.01 & $0.90(0.35,2.32)$ & 0.82 & $1.11(0.41,3.02)$ & 0.83 \\
\hline & T3 & $0.65(0.35,1.22)$ & 0.18 & $0.64(0.33,1.23)$ & 0.18 & $0.75(0.27,2.06)$ & 0.57 & $0.82(0.28,2.36)$ & 0.72 \\
\hline \multirow{2}{*}{ Sexual desire/activity change } & T1 & 1 & 1 & 1 & 1 & 1 & 1 & 1 & 1 \\
\hline & T3 & $1.07(0.66,1.74)$ & 0.78 & $1.06(0.65,1.73)$ & 0.81 & $0.75(0.43,1.29)$ & 0.3 & $0.81(0.46,1.44)$ & 0.47 \\
\hline
\end{tabular}

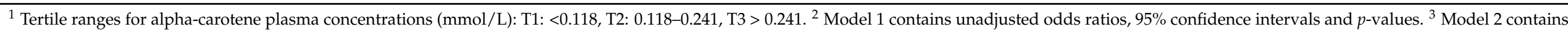
odds ratios, $95 \%$ confidence intervals and p-values adjusted for age, ethnicity, log-transformed BMI, plasma triglycerides levels, physical activity level and use of analgesics.

Table 5. Unadjusted and adjusted Odds Ratios and 95\% CIs for the association between tertiles of plasma retinol and premenstrual symptoms.

\begin{tabular}{|c|c|c|c|c|c|c|c|c|c|}
\hline \multirow{2}{*}{ Symptom } & \multirow{2}{*}{ Tertiles $^{1}$} & \multicolumn{4}{|c|}{ Mild vs. None } & \multicolumn{4}{|c|}{ Moderate/Severe vs. None } \\
\hline & & OR $(95 \% \mathrm{CI})^{2}$ & $p^{2}$ & OR $(95 \% \mathrm{CI})^{3}$ & $p^{3}$ & OR $(95 \% \mathrm{CI})^{2}$ & $p^{2}$ & OR $(95 \% \mathrm{CI})^{3}$ & $p^{3}$ \\
\hline \multirow{3}{*}{ Acne/skin blemish } & T1 & 1 & 1 & 1 & 1 & 1 & 1 & 1 & 1 \\
\hline & T2 & $1.30(0.83,2.04)$ & 0.25 & $1.37(0.86,2.19)$ & 0.18 & $0.88(0.49,1.59)$ & 0.68 & $0.81(0.44,1.48)$ & 0.49 \\
\hline & T3 & $0.84(0.53,1.34)$ & 0.47 & $0.83(0.51,1.36)$ & 0.47 & $1.35(0.79,2.31)$ & 0.27 & $1.20(0.69,2.12)$ & 0.51 \\
\hline \multirow{3}{*}{ Desire to be alone } & T1 & 1 & 1 & 1 & 1 & 1 & 1 & 1 & 1 \\
\hline & $\mathrm{T} 2$ & $1.44(0.88,2.35)$ & 0.14 & $1.48(0.90,2.45)$ & 0.12 & $1.76(0.92,3.38)$ & 0.08 & $1.63(0.83,3.18)$ & 0.15 \\
\hline & $\mathrm{T} 3$ & $1.08(0.66,1.80)$ & 0.75 & $1.20(0.71,2.04)$ & 0.49 & $1.63(0.85,3.11)$ & 0.14 & $1.57(0.80,3.10)$ & 0.19 \\
\hline \multirow{2}{*}{ Anxiety/tension/nervousness } & $\mathrm{T} 1$ & 1 & 1 & 1 & 1 & 1 & 1 & 1 & 1 \\
\hline & T2 & $1.11(0.70,1.78)$ & 0.06 & $0.95(0.59,1.55)$ & 0.85 & $0.61(0.32,1.19)$ & 0.15 & $1.53(0.79,2.99)$ & 0.21 \\
\hline \multirow{3}{*}{ Increased appetite/food cravings } & $\mathrm{T} 1$ & 1 & 1 & 1 & 1 & 1 & 1 & 1 & 1 \\
\hline & $\mathrm{T} 2$ & $1.03(0.62,1.70)$ & 0.91 & $0.98(0.59,1.64)$ & 0.95 & $1.19(0.73,1.93)$ & 0.49 & $1.19(0.73,1.96)$ & 0.48 \\
\hline & $\mathrm{T} 3$ & $1.29(0.78,2.13)$ & 0.32 & $1.19(0.70,2.01)$ & 0.52 & $1.44(0.88,2.36)$ & 0.14 & $1.46(0.88,2.44)$ & 0.14 \\
\hline \multirow{3}{*}{ Bloating/swelling/breast tenderness } & $\mathrm{T} 1$ & 1 & 1 & 1 & 1 & 1 & 1 & 1 & 1 \\
\hline & $\mathrm{T} 2$ & $1.25(0.76,2.07)$ & 0.38 & $1.27(0.76,2.12)$ & 0.35 & $1.27(0.74,2.16)$ & 0.37 & $1.17(0.68,2.01)$ & 0.57 \\
\hline & $\mathrm{T} 3$ & $0.97(0.59,1.60)$ & 0.91 & $0.96(0.57,1.61)$ & 0.86 & $1.27(0.76,2.12)$ & 0.37 & $1.17(0.68,2.00)$ & 0.57 \\
\hline \multirow{3}{*}{ Clumsiness } & $\mathrm{T} 1$ & 1 & 1 & 1 & 1 & 1 & 1 & 1 & 1 \\
\hline & $\mathrm{T} 2$ & $0.75(0.40,1.42)$ & 0.38 & $0.72(0.37,1.38)$ & 0.32 & $1.73(0.50,6.02)$ & 0.39 & $1.44(0.40,5.19)$ & 0.58 \\
\hline & T3 & $0.99(0.55,1.83)$ & 0.99 & $0.96(0.51,1.81)$ & 0.91 & $2.60(0.80,8.47)$ & 0.11 & $2.21(0.65,7.56)$ & 0.2 \\
\hline
\end{tabular}


Table 5. Cont.

\begin{tabular}{|c|c|c|c|c|c|c|c|c|c|}
\hline \multirow{2}{*}{ Symptom } & \multirow{2}{*}{ Tertiles $^{1}$} & \multicolumn{4}{|c|}{ Mild vs. None } & \multicolumn{4}{|c|}{ Moderate/Severe vs. None } \\
\hline & & OR $(95 \% \text { CI })^{2}$ & $p^{2}$ & OR $(95 \% \mathrm{CI})^{3}$ & $p^{3}$ & OR $(95 \% \mathrm{CI})^{2}$ & $p^{2}$ & OR $(95 \% \text { CI })^{3}$ & $p^{3}$ \\
\hline \multirow{3}{*}{$\begin{array}{l}\text { Confusion/difficulty } \\
\text { concentrating/forgetfulness }\end{array}$} & $\mathrm{T} 1$ & 1 & 1 & 1 & 1 & 1 & 1 & 1 & 1 \\
\hline & $\mathrm{T} 2$ & $0.84(0.48,1.45)$ & 0.54 & $0.83(0.47,1.46)$ & 0.52 & $0.68(0.29,1.58)$ & 0.37 & $0.58(0.24,1.39)$ & 0.22 \\
\hline & $\mathrm{T} 3$ & $0.75(0.42,1.32)$ & 0.31 & $0.76(0.41,1.38)$ & 0.36 & $1.34(0.65,2.77)$ & 0.43 & $1.26(0.59,2.72)$ & 0.55 \\
\hline \multirow{3}{*}{ Cramps } & $\mathrm{T} 1$ & 1 & 1 & 1 & 1 & 1 & 1 & 1 & 1 \\
\hline & $\mathrm{T} 2$ & $0.92(0.53,1.61)$ & 0.78 & $0.84(0.47,1.49)$ & 0.55 & $0.94(0.55,1.60)$ & 0.82 & $0.82(0.47,1.43)$ & 0.48 \\
\hline & T3 & $0.83(0.48,1.44)$ & 0.51 & $0.71(0.40,1.27)$ & 0.25 & $0.74(0.44,1.25)$ & 0.26 & $0.62(0.35,1.09)$ & 0.09 \\
\hline \multirow{3}{*}{ Depression } & T1 & 1 & 1 & 1 & 1 & 1 & 1 & 1 & 1 \\
\hline & $\mathrm{T} 2$ & $1.82(1.05,3.14)$ & 0.03 & $1.67(0.95,2.93)$ & 0.07 & $1.37(0.38,2.78)$ & 0.38 & $1.370 .66,2.81)$ & 0.4 \\
\hline & $\mathrm{T} 3$ & $1.30(0.73,2.31)$ & 0.37 & $1.08(0.59,1.97)$ & 0.8 & $1.50(0.76,2.98)$ & 0.25 & $1.64(0.80,3.36)$ & 0.18 \\
\hline \multirow{3}{*}{ Fatigue } & $\mathrm{T} 1$ & 1 & 1 & 1 & 1 & 1 & 1 & 1 & 1 \\
\hline & $\mathrm{T} 2$ & $0.79(0.50,1.27)$ & 0.33 & $0.82(0.50,1.32)$ & 0.41 & $0.94(0.56,1.59)$ & 0.81 & $0.95(0.55,1.63)$ & 0.85 \\
\hline & T3 & $0.72(0.45,1.16)$ & 0.17 & $0.77(0.47,1.26)$ & 0.29 & $0.96(0.57,1.62)$ & 0.88 & $1.08(0.63,1.86)$ & 0.78 \\
\hline \multirow{3}{*}{ Headaches } & T1 & 1 & 1 & 1 & 1 & 1 & 1 & 1 & 1 \\
\hline & $\mathrm{T} 2$ & $1.05(0.61,1.83)$ & 0.85 & $0.91(0.52,1.61)$ & 0.75 & $1.08(0.53,2.24)$ & 0.82 & $0.84(0.39,1.78)$ & 0.64 \\
\hline & $\mathrm{T} 3$ & $0.86(0.49,1.52)$ & 0.61 & $0.82(0.45,1.51)$ & 0.53 & $1.12(0.55,2.28)$ & 0.76 & $0.88(0.41,1.87)$ & 0.74 \\
\hline \multirow[b]{2}{*}{ Insomnia } & $\mathrm{T} 1$ & 1 & 1 & 1 & 1 & 1 & 1 & 1 & 1 \\
\hline & $\mathrm{T} 2$ & $0.95(0.48,1.88)$ & 0.89 & $0.91(0.45,1.85)$ & 0.8 & $1.20(0.36,4.04)$ & 0.76 & $1.10(0.32,3.85)$ & 0.87 \\
\hline \multirow{3}{*}{$\begin{array}{l}\text { Mood swings/crying } \\
\text { easily/irritability/angry outbursts }\end{array}$} & $\mathrm{T} 1$ & 1 & 1 & 1 & 1 & 1 & 1 & 1 & 1 \\
\hline & $\mathrm{T} 2$ & $0.72(0.43,1.20)$ & 0.2 & $0.79(0.47,1.34)$ & 0.38 & $1.21(0.71,2.04)$ & 0.48 & $1.18(0.70,2.02)$ & 0.53 \\
\hline & $\mathrm{T} 3$ & $0.91(0.54,1.52)$ & 0.71 & $1.06(0.62,1.83)$ & 0.82 & $1.41(0.83,2.39)$ & 0.21 & $1.47(0.84,2.56)$ & 0.18 \\
\hline \multirow{3}{*}{ Nausea } & T1 & 1 & 1 & 1 & 1 & 1 & 1 & 1 & 1 \\
\hline & $\mathrm{T} 2$ & $1.04(0.54,2.01)$ & 0.91 & $0.92(0.47,1.82)$ & 0.82 & $1.91(0.69,5.28)$ & 0.21 & $1.52(0.52,4.41)$ & 0.44 \\
\hline & $\mathrm{T} 3$ & $0.91(0.46,1.78)$ & 0.77 & $0.78(0.38,1.58)$ & 0.49 & $1.34(0.45,3.96)$ & 0.59 & $1.16(0.37,3.62)$ & 0.8 \\
\hline \multirow{3}{*}{ Sexual desire/activity change } & $\mathrm{T} 1$ & 1 & 1 & 1 & 1 & 1 & 1 & 1 & 1 \\
\hline & $\mathrm{T} 2$ & $1.12(0.70,1.79)$ & 0.64 & $1.10(0.69,1.79)$ & 0.67 & $2.35(1.31,4.23)$ & 0.004 & $2.17(1.17,4.01)$ & 0.014 \\
\hline & $\mathrm{T} 3$ & $1.40(0.89,2.20)$ & 0.14 & $1.41(0.88,2.27)$ & 0.15 & $2.52(0.81,2.83)$ & 0.19 & $1.41(0.73,2.75)$ & 0.31 \\
\hline
\end{tabular}

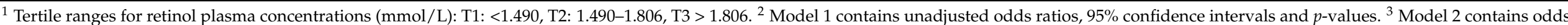

ratios, 95\% confidence intervals and $p$-values adjusted for age, ethnicity, log-transformed BMI, plasma triglycerides levels, physical activity level and use of analgesics. 
Table 6. Unadjusted and adjusted Odds Ratios and 95\% CIs for the association between tertiles of plasma lutein and premenstrual symptoms.

\begin{tabular}{|c|c|c|c|c|c|c|c|c|c|}
\hline \multirow{2}{*}{ Symptom } & \multirow[b]{2}{*}{ Tertiles $^{1}$} & \multicolumn{4}{|c|}{ Mild vs. None } & \multicolumn{4}{|c|}{ Moderate/Severe vs. None } \\
\hline & & OR $(95 \% \mathrm{CI})^{2}$ & $p^{2}$ & OR $(95 \% \mathrm{CI})^{3}$ & $p^{3}$ & OR $(95 \% \mathrm{CI})^{2}$ & $p^{2}$ & OR $(95 \% \mathrm{CI})^{3}$ & $p^{3}$ \\
\hline \multirow{3}{*}{ Acne/skin blemish } & $\mathrm{T} 1$ & 1 & 1 & 1 & 1 & 1 & 1 & 1 & 1 \\
\hline & $\mathrm{T} 2$ & $0.95(0.60,1.50)$ & 0.82 & $0.95(0.59,1.53)$ & 0.84 & $0.95(0.55,1.65)$ & 0.85 & $1.01(0.57,1.79)$ & 0.97 \\
\hline & $\mathrm{T} 3$ & $1.10(0.70,1.74)$ & 0.68 & $1.07(0.66,1.73)$ & 0.78 & $1.05(0.61,1.84)$ & 0.85 & $1.12(0.63,2.00)$ & 0.71 \\
\hline \multirow{3}{*}{ Desire to be alone } & $\mathrm{T} 1$ & 1 & 1 & 1 & 1 & 1 & 1 & 1 & 1 \\
\hline & $\mathrm{T} 2$ & $0.98(0.60,1.59)$ & 0.93 & $0.97(0.59,1.59)$ & 0.9 & $0.71(0.38,1.34)$ & 0.29 & $0.78(0.41,1.50)$ & 0.15 \\
\hline & T3 & $0.79(0.48,1.30)$ & 0.35 & $0.77(0.46,1.29)$ & 0.31 & $0.86(0.47,1.58)$ & 0.64 & $1.05(0.56,1.97)$ & 0.19 \\
\hline \multirow{2}{*}{ Anxiety/tension/nervousness } & $\mathrm{T} 1$ & 1 & 1 & 1 & 1 & 1 & 1 & 1 & 1 \\
\hline & $\mathrm{T} 3$ & $0.83(0.52,1.35)$ & 0.46 & $0.76(0.46,1.25)$ & 0.29 & $1.05(0.56,1.99)$ & 0.87 & $1.02(0.52,1.98)$ & 0.96 \\
\hline \multirow{3}{*}{ Increased appetite/food cravings } & $\mathrm{T} 1$ & 1 & 1 & 1 & 1 & 1 & 1 & 1 & 1 \\
\hline & $\mathrm{T} 2$ & $0.85(0.52,1.41)$ & 0.53 & $0.92(0.55,1.53)$ & 0.74 & $0.87(0.50,1.20)$ & 0.21 & $0.76(0.46,1.25)$ & 0.28 \\
\hline & $\mathrm{T} 3$ & $0.74(0.53,1.45)$ & 0.6 & $0.99(0.58,1.68)$ & 0.97 & $0.93(0.57,1.51)$ & 0.76 & $0.98(0.59,1.62)$ & 0.94 \\
\hline \multirow{3}{*}{ Bloating/swelling/breast tenderness } & $\mathrm{T} 1$ & 1 & 1 & 1 & 1 & 1 & 1 & 1 & 1 \\
\hline & $\mathrm{T} 2$ & $0.95(0.57,1.57)$ & 0.84 & $0.95(0.57,1.60)$ & 0.86 & $0.93(0.55,1.57)$ & 0.79 & $1.04(0.61,1.78)$ & 0.9 \\
\hline & $\mathrm{T} 3$ & $0.83(0.50,1.38)$ & 0.47 & $0.83(0.49,1.39)$ & 0.48 & $0.86(0.51,1.45)$ & 0.58 & $0.98(0.57,1.70)$ & 0.96 \\
\hline \multirow[b]{2}{*}{ Clumsiness } & $\mathrm{T} 1$ & 1 & 1 & 1 & 1 & 1 & 1 & 1 & 1 \\
\hline & $\mathrm{T} 2$ & $0.62(0.32,1.18)$ & 0.15 & $0.55(0.28,1.08)$ & 0.08 & $0.83(0.29,2.33)$ & 0.72 & $0.82(0.28,2.40)$ & 0.71 \\
\hline \multirow{3}{*}{$\begin{array}{l}\text { Confusion/difficulty } \\
\text { concentrating/forgetfulness }\end{array}$} & $\mathrm{T} 1$ & 1 & 1 & 1 & 1 & 1 & 1 & 1 & 1 \\
\hline & $\mathrm{T} 2$ & $0.85(0.48,1.52)$ & 0.59 & $0.74(0.41,1.34)$ & 0.32 & $1.07(0.47,2.42)$ & 0.87 & $1.04(0.45,2.41)$ & 0.93 \\
\hline & $\mathrm{T} 3$ & $1.09(0.63,1.91)$ & 0.75 & $0.87(0.54,1.72)$ & 0.91 & $1.59(0.74,3.42)$ & 0.24 & $1.59(0.71,3.56)$ & 0.25 \\
\hline \multirow{3}{*}{ Cramps } & T1 & 1 & 1 & 1 & 1 & 1 & 1 & 1 & 1 \\
\hline & T2 & $1.16(0.65,2.08)$ & 0.61 & $1.320 .73,2.38)$ & 0.36 & $1.00(0.55,1.60)$ & 0.99 & $1.24(0.71,2.20)$ & 0.45 \\
\hline & $\mathrm{T} 3$ & $0.76(0.44,2.08)$ & 0.32 & $0.88(0.50,1.54)$ & 0.65 & $0.48(0.29,0.81)$ & 0.006 & $0.62(0.35,1.07)$ & 0.08 \\
\hline \multirow{3}{*}{ Depression } & $\mathrm{T} 1$ & 1 & 1 & 1 & 1 & 1 & 1 & 1 & 1 \\
\hline & $\mathrm{T} 2$ & $0.85(0.50,1.46)$ & 0.56 & $0.83(0.47,1.44)$ & 0.5 & $0.86(0.43,1.73)$ & 0.68 & $0.88(0.72,0.43)$ & 0.72 \\
\hline & $\mathrm{T} 3$ & $0.91(0.53,1.56)$ & 0.74 & $0.90(0.52,1.58)$ & 0.71 & $1.10(0.57,2.15)$ & 0.77 & $1.12(0.56,2.26)$ & 0.74 \\
\hline \multirow{3}{*}{ Fatigue } & $\mathrm{T} 1$ & 1 & 1 & 1 & 1 & 1 & 1 & 1 & 1 \\
\hline & $\mathrm{T} 2$ & $0.89(0.55,1.42)$ & 0.62 & $0.86(0.53,1.40)$ & 0.54 & $1.15(0.68,1.94)$ & 0.6 & $1.11(0.65,1.90)$ & 0.69 \\
\hline & $\mathrm{T} 3$ & $0.98(0.61,1.56)$ & 0.92 & $0.94(0.58,1.52)$ & 0.8 & $1.13(0.67,1.91)$ & 0.66 & $1.08(0.63,1.88)$ & 0.77 \\
\hline \multirow{3}{*}{ Headaches } & $\mathrm{T} 1$ & 1 & 1 & 1 & 1 & 1 & 1 & 1 & 1 \\
\hline & $\mathrm{T} 2$ & $0.74(0.42,1.31)$ & 0.3 & $0.83(0.46,1.49)$ & 0.53 & $0.84(0.41,1.71)$ & 0.63 & $0.85(0.41,1.79)$ & 0.68 \\
\hline & $\mathrm{T} 3$ & $0.93(0.54,1.60)$ & 0.78 & $1.12(0.63,1.99)$ & 0.71 & $0.93(0.46,1.88)$ & 0.84 & $0.98(0.47,2.05)$ & 0.95 \\
\hline \multirow{3}{*}{ Insomnia } & $\mathrm{T} 1$ & 1 & 1 & 1 & 1 & 1 & 1 & 1 & 1 \\
\hline & $\mathrm{T} 2$ & $0.50(0.24,1.04)$ & 0.06 & $0.54(0.25,1.14)$ & 0.1 & $1.93(0.57,6.53)$ & 0.29 & $2.36(0.67,8.35)$ & 0.18 \\
\hline & T3 & $0.72(0.37,1.39)$ & 0.32 & $0.77(0.38,1.56)$ & 0.47 & $0.97(0.24,3.94)$ & 0.97 & $1.23(0.28,5.35)$ & 0.78 \\
\hline
\end{tabular}


Table 6. Cont.

\begin{tabular}{|c|c|c|c|c|c|c|c|c|c|}
\hline \multirow{2}{*}{ Symptom } & \multirow[b]{2}{*}{ Tertiles ${ }^{1}$} & \multicolumn{4}{|c|}{ Mild vs. None } & \multicolumn{4}{|c|}{ Moderate/Severe vs. None } \\
\hline & & OR $(95 \% \mathrm{CI})^{2}$ & $p^{2}$ & OR $(95 \% \mathrm{CI})^{3}$ & $p^{3}$ & OR $(95 \% \mathrm{CI})^{2}$ & $p^{2}$ & OR $(95 \% \mathrm{CI})^{3}$ & $p^{3}$ \\
\hline \multirow{3}{*}{$\begin{array}{l}\text { Mood swings/crying } \\
\text { easily/irritability/angry outbursts }\end{array}$} & $\mathrm{T} 1$ & 1 & 1 & 1 & 1 & 1 & 1 & 1 & 1 \\
\hline & $\mathrm{T} 2$ & $0.53(0.32,0.88)$ & 0.015 & $0.49(0.28,0.83)$ & 0.008 & $0.85(0.50,1.43)$ & 0.53 & $0.89(0.52,1.53)$ & 0.68 \\
\hline & T3 & $0.74(0.44,1.25)$ & 0.27 & $0.66(0.38,1.13)$ & 0.13 & $1.02(0.60,1.74)$ & 0.94 & $1.08(0.62,1.89)$ & 0.78 \\
\hline \multirow{3}{*}{ Nausea } & T1 & 1 & 1 & 1 & 1 & 1 & 1 & 1 & 1 \\
\hline & $\mathrm{T} 2$ & $0.87(0.45,1.69)$ & 0.69 & $0.94(0.48,1.85)$ & 0.85 & $0.67(00.28,1.61)$ & 0.37 & $0.79(0.31,1.97)$ & 0.61 \\
\hline & $\mathrm{T} 3$ & $0.79(0.41,1.55)$ & 0.5 & $0.86(0.42,1.72)$ & 0.66 & $0.21(0.06,0.76)$ & 0.017 & $0.25(0.05,0.93)$ & 0.04 \\
\hline \multirow{3}{*}{ Sexual desire/activity change } & T1 & 1 & 1 & 1 & 1 & 1 & 1 & 1 & 1 \\
\hline & $\mathrm{T} 2$ & $0.83(0.52,1.32)$ & 0.43 & $0.87(0.54,1.39)$ & 0.55 & $0.94(0.54,1.64)$ & 0.82 & $1.17(0.65,2.13)$ & 0.59 \\
\hline & $\mathrm{T} 3$ & $0.90(0.57,1.42)$ & 0.64 & $0.92(0.58,1.48)$ & 0.74 & $0.76(0.42,1.36)$ & 0.35 & $0.99(0.53,1.85)$ & 0.98 \\
\hline
\end{tabular}

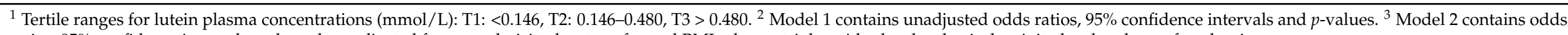
ratios, $95 \%$ confidence intervals and $p$-values adjusted for age, ethnicity, log-transformed BMI, plasma triglycerides levels, physical activity level and use of analgesics.

Table 7. Unadjusted and adjusted Odds Ratios and 95\% CIs for the association between tertiles of plasma zeaxanthin and premenstrual symptoms.

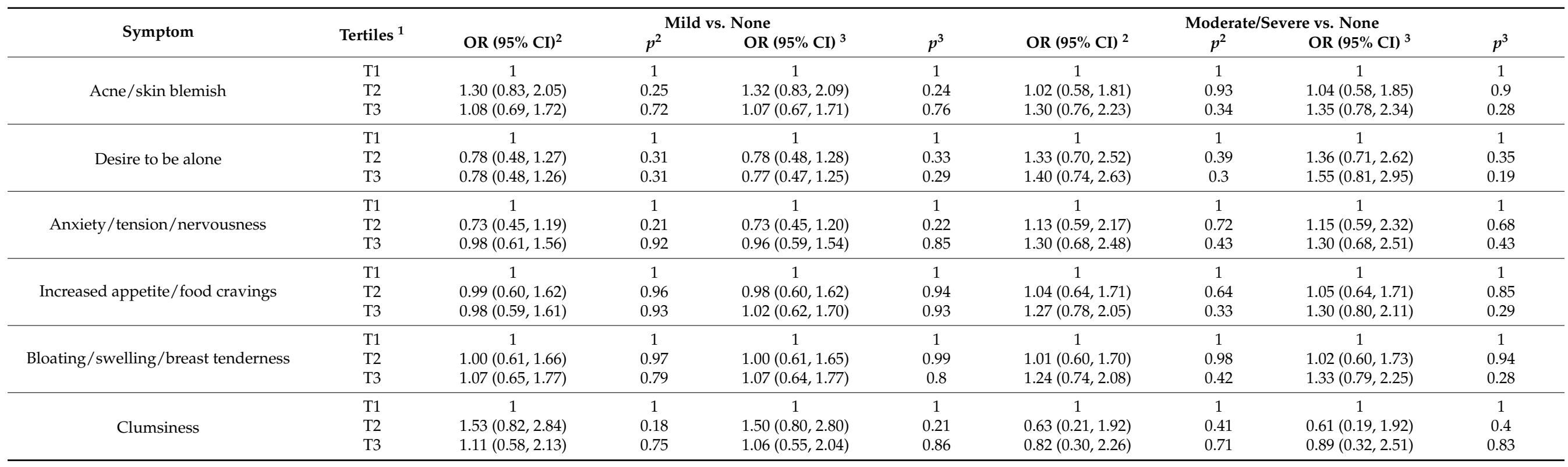


Table 7. Cont.

\begin{tabular}{|c|c|c|c|c|c|c|c|c|c|}
\hline \multirow{2}{*}{ Symptom } & \multirow{2}{*}{ Tertiles $^{1}$} & \multicolumn{4}{|c|}{ Mild vs. None } & \multicolumn{4}{|c|}{ Moderate/Severe vs. None } \\
\hline & & OR $(95 \% \mathrm{CI})^{2}$ & $p^{2}$ & OR $(95 \% \text { CI })^{3}$ & $p^{3}$ & OR $(95 \% \text { CI })^{2}$ & $p^{2}$ & OR $(95 \% \mathrm{CI})^{3}$ & $p^{3}$ \\
\hline \multirow{3}{*}{$\begin{array}{l}\text { Confusion/difficulty } \\
\text { concentrating/forgetfulness }\end{array}$} & $\mathrm{T} 1$ & 1 & 1 & 1 & 1 & 1 & 1 & 1 & 1 \\
\hline & $\mathrm{T} 2$ & $0.95(0.54,1.68)$ & 0.87 & $0.93(0.52,1.65)$ & 0.8 & $1.18(0.54,2.60)$ & 0.68 & $1.20(0.54,2.68)$ & 0.65 \\
\hline & $\mathrm{T} 3$ & $1.01(0.58,1.76)$ & 0.97 & $0.94(0.54,1.66)$ & 0.84 & $1.33(0.62,2.86)$ & 0.47 & $1.34(0.62,2.93)$ & 0.46 \\
\hline \multirow{3}{*}{ Cramps } & T1 & 1 & 1 & 1 & 1 & 1 & 1 & 1 & 1 \\
\hline & $\mathrm{T} 2$ & $0.65(0.38,1.14)$ & 0.13 & $0.63(0.36,1.11)$ & 0.11 & $0.59(0.35,1.01)$ & 0.054 & $0.57(0.33,0.99)$ & 0.044 \\
\hline & T3 & $0.78(0.45,1.36)$ & 0.38 & $0.81(0.46,1.42)$ & 0.46 & $0.73(0.43,1.24)$ & 0.24 & $0.79(0.45,1.37)$ & 0.4 \\
\hline \multirow{3}{*}{ Depression } & $\mathrm{T} 1$ & 1 & 1 & 1 & 1 & 1 & 1 & 1 & 1 \\
\hline & $\mathrm{T} 2$ & $1.39(0.82,2.36)$ & 0.23 & $1.36(0.79,2.32)$ & 0.27 & $1.42(0.70,2.89)$ & 0.33 & $1.42(0.70,2.90)$ & 0.33 \\
\hline & T3 & $0.99(0.57,1.73)$ & 0.99 & $0.98(0.56,1.72)$ & 0.95 & $1.51(0.76,3.00)$ & 0.24 & $1.53(0.77,3.05)$ & 0.23 \\
\hline \multirow{3}{*}{ Fatigue } & T1 & 1 & 1 & 1 & 1 & 1 & 1 & 1 & 1 \\
\hline & $\mathrm{T} 2$ & $1.15(0.72,1.84)$ & 0.57 & $1.16(0.72,1.87)$ & 0.54 & $1.15(0.66,1.88)$ & 0.68 & $1.14(0.67,1.92)$ & 0.63 \\
\hline & $\mathrm{T} 3$ & $1.13(0.70,1.80)$ & 0.62 & $1.12(0.70,1.80)$ & 0.64 & $1.13(0.67,1.88)$ & 0.65 & $1.11(0.66,1.87)$ & 0.7 \\
\hline \multirow{3}{*}{ Headaches } & T1 & 1 & 1 & 1 & 1 & 1 & 1 & 1 & 1 \\
\hline & $\mathrm{T} 2$ & $0.72(0.41,1.27)$ & 0.25 & $0.71(0.40,1.27)$ & 0.24 & $1.10(0.55,2.19)$ & 0.78 & $1.06(0.53,2.15)$ & 0.86 \\
\hline & T3 & $0.90(0.53,1.54)$ & 0.7 & $0.93(0.54,1.61)$ & 0.79 & $0.79(0.38,1.65)$ & 0.53 & $0.79(0.37,1.69)$ & 0.55 \\
\hline \multirow[b]{2}{*}{ Insomnia } & $\mathrm{T} 1$ & 1 & 1 & 1 & 1 & 1 & 1 & 1 & 1 \\
\hline & T2 & $1.07(0.55,2.10)$ & 0.84 & $1.09(0.55,2.16)$ & 0.8 & $0.40(0.10,1.54)$ & 0.18 & $0.41(0.10,1.58)$ & 0.19 \\
\hline \multirow{3}{*}{$\begin{array}{c}\text { Mood swings/crying } \\
\text { easily/irritability/angry outbursts }\end{array}$} & $\mathrm{T} 1$ & 1 & 1 & 1 & 1 & 1 & 1 & 1 & 1 \\
\hline & $\mathrm{T} 2$ & $0.74(0.44,1.23)$ & 0.25 & $0.74(0.44,1.25)$ & 0.26 & $0.91(0.54,1.54)$ & 0.73 & $0.92(0.54,1.55)$ & 0.76 \\
\hline & T3 & $0.81(0.49,1.36$ & 0.43 & $0.77(0.46,1.30)$ & 0.34 & $0.96(0.57,1.62)$ & 0.88 & $0.98(0.58,1.65)$ & 0.93 \\
\hline \multirow{3}{*}{ Nausea } & T1 & 1 & 1 & 1 & 1 & 1 & 1 & 1 & 1 \\
\hline & $\mathrm{T} 2$ & $0.83(0.42,1.64)$ & 0.59 & $0.84(0.42,1.70)$ & 0.63 & $1.40(0.51,3.84)$ & 0.52 & $1.47(0.52,4.17)$ & 0.47 \\
\hline & $\mathrm{T} 3$ & $1.08(0.57,2.05)$ & 0.82 & $1.18(0.61,2.28)$ & 0.62 & $1.39(0.50,3.81)$ & 0.53 & $1.63(0.57,4.67)$ & 0.36 \\
\hline \multirow{3}{*}{ Sexual desire/activity change } & $\mathrm{T} 1$ & 1 & 1 & 1 & 1 & 1 & 1 & 1 & 1 \\
\hline & $\mathrm{T} 2$ & $1.14(0.72,1.81)$ & 0.57 & $1.15(0.72,1.82)$ & 0.56 & $1.36(0.77,2.40)$ & 0.29 & $1.45(0.80,2.62)$ & 0.22 \\
\hline & T3 & $1.01(0.64,1.59)$ & 0.96 & $1.02(0.65,1.61)$ & 0.93 & $1.03(0.57,1.84)$ & 0.93 & $1.20(0.66,2.19)$ & 0.56 \\
\hline
\end{tabular}

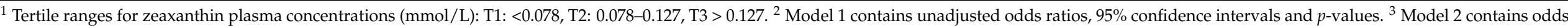

ratios, $95 \%$ confidence intervals and $p$-values adjusted for age, ethnicity, log-transformed BMI, plasma triglycerides levels, physical activity level and use of analgesics. 
Table 8. Unadjusted and adjusted Odds Ratios and 95\% CIs for the association between tertiles of plasma trans-lycopene and premenstrual symptoms.

\begin{tabular}{|c|c|c|c|c|c|c|c|c|c|}
\hline \multirow{2}{*}{ Symptom } & \multirow{2}{*}{ Tertiles ${ }^{1}$} & \multicolumn{4}{|c|}{ Mild vs. None } & \multicolumn{4}{|c|}{ Moderate/Severe vs. None } \\
\hline & & OR $(95 \% \text { CI })^{2}$ & $p^{2}$ & OR $(95 \% \mathrm{CI})^{3}$ & $p^{3}$ & OR $(95 \% \mathrm{CI})^{2}$ & $p^{2}$ & OR $(95 \% \mathrm{CI})^{3}$ & $p^{3}$ \\
\hline \multirow{3}{*}{ Acne/skin blemish } & $\mathrm{T} 1$ & 1 & 1 & 1 & 1 & 1 & 1 & 1 & 1 \\
\hline & $\mathrm{T} 2$ & $1.34(0.84,2.13)$ & 0.22 & $1.32(0.82,2.12)$ & 0.25 & $1.79(1.03,3.09)$ & 0.038 & $1.73(1.00,3.03)$ & 0.053 \\
\hline & $\mathrm{T} 3$ & $1.03(0.66 \mathrm{~m} 1.62)$ & 0.9 & $1.07(0.67,1.72)$ & 0.77 & $0.91(0.51,1.62)$ & 0.75 & $0.94(0.52,1.70)$ & 0.84 \\
\hline \multirow{3}{*}{ Desire to be alone } & T1 & 1 & 1 & 1 & 1 & 1 & 1 & 1 & 1 \\
\hline & $\mathrm{T} 2$ & $0.77(0.47,1.26)$ & 0.3 & $0.78(0.47,1.29)$ & 0.33 & $1.12(0.60,2.09)$ & 0.39 & $1.12(0.59,2.11)$ & 0.73 \\
\hline & $\mathrm{T} 3$ & $0.94(0.58,1.52)$ & 0.79 & $0.96(0.58,1.57)$ & 0.86 & $1.09(0.58,2.06)$ & 0.3 & $1.03(0.54,1.98)$ & 0.93 \\
\hline \multirow{2}{*}{ Anxiety/tension/nervousness } & $\mathrm{T} 1$ & 1 & 1 & 1 & 1 & 1 & 1 & 1 & 1 \\
\hline & T3 & $1.01(0.62,1.65)$ & 0.96 & $1.13(0.68,1.86)$ & 0.64 & $1.23(0.65,2.32)$ & 0.52 & $1.34(0.70,2.59)$ & 0.38 \\
\hline \multirow{3}{*}{ Increased appetite/food cravings } & $\mathrm{T} 1$ & 1 & 1 & 1 & 1 & 1 & 1 & 1 & 1 \\
\hline & $\mathrm{T} 2$ & $1.27(0.77,2.10)$ & 0.36 & $1.26(0.66,1.88)$ & 0.38 & $1.07(0.66,1.74)$ & 0.78 & $1.08(0.66,1.77)$ & 0.75 \\
\hline & $\mathrm{T} 3$ & $1.18(0.71,1.96)$ & 0.52 & $1.11(0.85,1.02)$ & 0.68 & $1.05(0.65,1.71)$ & 0.83 & $1.07(0.65,1.75)$ & 0.8 \\
\hline \multirow{3}{*}{ Bloating/swelling/breast tenderness } & T1 & 1 & 1 & 1 & 1 & 1 & 1 & 1 & 1 \\
\hline & $\mathrm{T} 2$ & $1.24(0.76,2.04)$ & 0.4 & $1.20(0.73,1.99)$ & 0.47 & $1.56(0.92,2.63)$ & 0.09 & $1.49(0.88,2.54)$ & 0.14 \\
\hline & $\mathrm{T} 3$ & $1.33(0.80,2.20)$ & 0.27 & $1.31(0.78,2.19)$ & 0.31 & $1.77(1.04,3.00)$ & 0.033 & $1.74(1.01,2.99)$ & 0.045 \\
\hline \multirow[b]{2}{*}{ Clumsiness } & T1 & 1 & 1 & 1 & 1 & 1 & 1 & 1 & 1 \\
\hline & $\mathrm{T} 2$ & $0.66(0.35,1.22)$ & 0.18 & $0.68(0.36,1.26)$ & 0.22 & $0.51(0.17,1.56)$ & 0.24 & $0.52(0.17,1.62)$ & 0.26 \\
\hline \multirow{3}{*}{$\begin{array}{c}\text { Confusion/difficulty } \\
\text { concentrating/forgetfulness }\end{array}$} & T1 & 1 & 1 & 1 & 1 & 1 & 1 & 1 & 1 \\
\hline & $\mathrm{T} 2$ & $1.09(0.62,1.92)$ & 0.76 & $1.20(0.68,2.14)$ & 0.53 & $0.77(0.37,1.60)$ & 0.48 & $0.77(0.37,1.63)$ & 0.5 \\
\hline & T3 & $0.96(0.54,1.70)$ & 0.88 & $1.11(0.61,2.01)$ & 0.73 & $0.59(0.27,1.28)$ & 0.18 & $0.62(0.28,1.40)$ & 0.25 \\
\hline \multirow{3}{*}{ Cramps } & $\mathrm{T} 1$ & 1 & 1 & 1 & 1 & 1 & 1 & 1 & 1 \\
\hline & $\mathrm{T} 2$ & $0.76(0.44,1.29)$ & 0.31 & $0.70(0.41,1.21)$ & 0.2 & $0.99(0.59,1.66)$ & 0.96 & $0.92(0.53,1.58)$ & 0.75 \\
\hline & T3 & $0.98(0.56,1.72)$ & 0.96 & $0.85(0.48,1.52)$ & 0.59 & $0.150(0.87,2.56)$ & 0.14 & $1.21(0.69,2.13)$ & 0.51 \\
\hline \multirow{3}{*}{ Depression } & T1 & 1 & 1 & 1 & 1 & 1 & 1 & 1 & 1 \\
\hline & $\mathrm{T} 2$ & $1.42(0.84,2.39)$ & 0.19 & $1.37(0.80,2.34)$ & 0.25 & $0.81(0.39,1.70)$ & 0.59 & $0.80(0.28,1.69)$ & 0.57 \\
\hline & $\mathrm{T} 3$ & $0.82(0.46,1.46)$ & 0.5 & $0.84(0.46,1.52)$ & 0.56 & $1.41(0.74,2.70)$ & 0.3 & $1.44(0.73,2.82)$ & 0.29 \\
\hline \multirow{3}{*}{ Fatigue } & T1 & 1 & 1 & 1 & 1 & 1 & 1 & 1 & 1 \\
\hline & $\mathrm{T} 2$ & $1.24(0.77,2.00)$ & 0.38 & $1.27(0.78,2.06)$ & 0.33 & $0.85(0.51,1.43)$ & 0.55 & $0.89(0.53,1.51)$ & 0.68 \\
\hline & T3 & $1.23(0.76,2.00)$ & 0.4 & $1.23(0.75,2.02)$ & 0.41 & $0.92(0.55,1.54)$ & 0.75 & $0.98(0.58,1.66)$ & 0.94 \\
\hline \multirow{3}{*}{ Headaches } & T1 & 1 & 1 & 1 & 1 & 1 & 1 & 1 & 1 \\
\hline & $\mathrm{T} 2$ & $0.87(0.49,1.54)$ & 0.63 & $0.87(0.48,1.56)$ & 0.64 & $1.31(0.66,2.62)$ & 0.44 & $1.26(0.62,2.56)$ & 0.53 \\
\hline & $\mathrm{T} 3$ & $1.16(0.67,2.00)$ & 0.6 & $1.12(0.63,1.97)$ & 0.7 & $0.89(0.42,1.90)$ & 0.77 & $0.86(0.39,1.87)$ & 0.7 \\
\hline \multirow{3}{*}{ Insomnia } & $\mathrm{T} 1$ & 1 & 1 & 1 & 1 & 1 & 1 & 1 & 1 \\
\hline & $\mathrm{T} 2$ & $0.93(0.46,1.87)$ & 0.85 & $0.97(0.48,1.98)$ & 0.94 & $0.99(0.24,4.02)$ & 0.99 & $1.00(0.24,4.09)$ & 0.99 \\
\hline & $\mathrm{T} 3$ & $0.97(0.48,1.95)$ & 0.93 & $1.06(0.51,2.18)$ & 0.88 & $2.05(0.61,6.95)$ & 0.25 & $2.03(0.58,7.16)$ & 0.27 \\
\hline
\end{tabular}


Table 8. Cont.

\begin{tabular}{|c|c|c|c|c|c|c|c|c|c|}
\hline \multirow{2}{*}{ Symptom } & \multirow{2}{*}{ Tertiles $^{1}$} & \multicolumn{4}{|c|}{ Mild vs. None } & \multicolumn{4}{|c|}{ Moderate/Severe vs. None } \\
\hline & & OR $(95 \% \mathrm{CI})^{2}$ & $p^{2}$ & OR $(95 \% \mathrm{CI})^{3}$ & $p^{3}$ & OR $(95 \% \mathrm{CI})^{2}$ & $p^{2}$ & OR $(95 \% \mathrm{CI})^{3}$ & $p^{3}$ \\
\hline \multirow{3}{*}{$\begin{array}{c}\text { Mood swings/crying } \\
\text { easily/irritability/angry outbursts }\end{array}$} & T1 & 1 & 1 & 1 & 1 & 1 & 1 & 1 & 1 \\
\hline & $\mathrm{T} 2$ & $1.08(0.65,1.80)$ & 0.76 & $1.10(0.66,1.86)$ & 0.71 & $1.32(0.78,2.25)$ & 0.3 & $1.31(0.77,2.23)$ & 0.32 \\
\hline & T3 & $0.88(0.53,1.47)$ & 0.64 & $0.92(0.54,1.56)$ & 0.75 & $1.26(0.75,2.12)$ & 0.39 & $1.27(0.74,2.16)$ & 0.39 \\
\hline \multirow{3}{*}{ Nausea } & $\mathrm{T} 1$ & 1 & 1 & 1 & 1 & 1 & 1 & 1 & 1 \\
\hline & $\mathrm{T} 2$ & $1.43(0.71,2.87)$ & 0.32 & $1.40(0.69,2.84)$ & 0.36 & $0.82(0.31,2.12)$ & 0.68 & $0.77(0.29,2.07)$ & 0.61 \\
\hline & $\mathrm{T} 3$ & $1.51(0.76,3.03)$ & 0.24 & $1.39(0.68,2.83)$ & 0.36 & $0.72(0.27,1.95)$ & 0.52 & $0.63(0.22,1.78)$ & 0.39 \\
\hline \multirow{2}{*}{ Sexual desire/activity change } & $\mathrm{T} 1$ & 1 & 1 & 1 & 1 & 1 & 1 & 1 & 1 \\
\hline & T3 & $1.15(0.72,1.82)$ & 0.56 & $1.18(0.73,1.90)$ & 0.49 & $1.72(0.95,3.10)$ & 0.07 & $1.54(0.83,2.87)$ & 0.56 \\
\hline
\end{tabular}

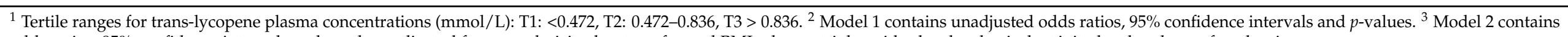
odds ratios, 95\% confidence intervals and $p$-values adjusted for age, ethnicity, log-transformed BMI, plasma triglycerides levels, physical activity level and use of analgesics. 


\section{Discussion}

The present cross-sectional study of 553 young women living in Canada examined the association between plasma carotenoids and retinol on the severity of 15 common premenstrual symptoms. While previous research on this topic assessed retinol alone [19-22], the current study included six different plasma carotenoids along with retinol to provide a more comprehensive understanding of the role of vitamin A and provitamin A carotenoids, which may have unique biological effects. Further, using serum concentrations as opposed to dietary intake allowed a more direct measure of exposure as serum values take into account the variability of an individual's metabolism. We observed a positive association between plasma beta-cryptoxanthin concentrations and increased appetite/food cravings during the premenstrual period, which remained significant after adjusting for potential confounders and accounting for multiple comparisons. No other plasma carotenoids or retinol were associated with the severity of the 15 common premenstrual symptoms examined in the present study after adjustments for multiple comparisons.

Provitamin A in the form of carotenoids, the brightly coloured pigments in most orange and yellow foods, is common in the diet and acts as a powerful antioxidant when in carotenoid form. Recent studies have shown that beta-cryptoxanthin, commonly found in squash, hot chilli peppers and tangerines, are absorbed more readily than other carotenoids, giving it a bioavailability from carotenoid-rich foods that is considerably higher than beta-carotene, which is traditionally considered to have the highest biological conversion activity and is used as a marker of provitamin A physiological status $[14,26]$. Once digested, beta-cryptoxanthin is absorbed through facilitated transport at low physiological concentrations [26]. Passive diffusion supplements this process with higher pharmacological doses. It then undergoes a biosynthetic conversion to retinol in the enterocytes; however, this conversion process is poorly understood [26]. Other common carotenoids include alphacarotene, zeaxanthin, and lutein. Vitamin A forming carotenoids, such as beta-carotene and beta-cryptoxanthin, are converted into retinol once in the body. However, due to genetic variability, humans have varying abilities to carry out this conversion process, and therefore, retinol is the most reliable marker of vitamin A [27]. While carotenoids function and have biological effects as precursors for vitamin A, they also function as antioxidants and, through these effects, may play a role in premenstrual symptoms [11].

Previous research suggests that antioxidants may decrease the incidence of certain chronic diseases because of their role in reducing oxidative damage and inflammation [13]. Carotenoids are amongst the strongest antioxidants, suggesting a potential ameliorating effect on premenstrual symptoms through this pathway [11]. Studies have shown that inflammatory markers, such as the interleukins 2, 4, 10 and 12, along with interferongamma, are positively associated with premenstrual symptom severity [12]. Elevated serum concentrations of high-sensitivity C-reactive protein (hs-CRP), a molecule elevated during conditions of inflammation, were associated with a score of menstrual symptoms, where symptoms were categorized into one of four groups: behaviour, mood, pain or physical symptoms [28]. Another cross-sectional study reproduced these results, showing a significant positive association between hs-CRP concentrations and the following premenstrual symptoms: mood swings, abdominal cramps/back pain, appetite cravings/weight gain/bloating and breast pain [29]. These results suggest that anti-inflammatory agents, such as antioxidants, may play a role in the prevention or treatment of some premenstrual symptoms.

Given the limited number of studies on carotenoids and premenstrual symptoms [19-22], the present study examined whether plasma carotenoid concentrations were associated with premenstrual symptoms in young women of reproductive age. Based on their role as antioxidants, it was hypothesized that carotenoids would be associated with a lower risk of premenstrual symptoms; however, circulating concentrations of several common carotenoids were not associated with any symptoms. Furthermore, beta-cryptoxanthin showed a positive association with an increased appetite and food cravings. This finding may be due, in part, to differences in the biological activity of different carotenoids. 
Carotenoids can be classified into two main categories: vitamin A forming carotenoids and non-vitamin A forming carotenoids [26]. Lycopene is an example of a non-vitamin A forming carotenoid, while beta-cryptoxanthin is an example of a vitamin A forming carotenoid and is converted to vitamin A once in the body. It is possible that our observations of increased severity of food cravings within the highest tertile of beta-cryptoxanthin is due to reverse causation, where women who experience moderate/severe food cravings during their menses increase their dietary consumption to meet their new hunger requirements, and in doing so, may transiently increase their intake of beta-cryptoxanthin containing foods.

Results from previous studies on vitamin A and premenstrual symptoms have been inconsistent. Frankel and colleagues recently evaluated the relationship between serum antioxidant vitamins, such as vitamins A, C and E, and PMS in 259 healthy women aged 18-49 in western New York, U.S.A [21]. There were no associations between serum vitamin A, measured using retinol as a biomarker, and the prevalence or the severity of PMS, a result that is consistent with the findings of the present study. Bahrami and colleagues also recently investigated the associations between serum fat-soluble vitamin concentrations, such as vitamin A and E, and PMS in 897 Iranian adolescents and found that serum vitamin A, measured by trans retinol, was inversely associated with the presence of PMS [22]. The discrepancy in results may potentially be due to the ethnic differences in the occurrence and severity of premenstrual symptoms, where a few observational studies have noted a higher prevalence of premenstrual symptoms in certain ethnic groups $[3,30]$.

While most of the research on this topic assesses retinol alone as a marker for vitamin A, one strength of the present study was that it compared seven different plasma carotenoids to provide a more comprehensive understanding of the role of vitamin A and the individual carotenoids. Further, using serum concentrations, as opposed to dietary intake, allowed a more accurate measure of exposure, as serum values take into account the variability of an individual's metabolism. The large amount of data collected in the present study population enabled us to adjust for a number of factors known to influence premenstrual symptoms and carotenoid levels, thereby reducing the likelihood of residual confounding. Lastly, this study represents a multi-ethnic population, whereas most other studies have focused on homogeneous groups of women.

The present study is not without its limitations. A retrospective questionnaire was used to report premenstrual symptom severities, which could result in over or underestimation of the prevalence and severities of some symptoms. The participants were also young adults recruited from a large urban university campus, so the results may not be representative of all menstruating females. Although the timing of blood sample collection for carotenoids was not particular to the luteal phase, it is not clear whether beta-cryptoxanthin levels differ during the menstrual cycle. A previous study assessed serum carotenoid variations across the menstrual cycle but did not measure beta-cryptoxanthin [31]. Findings from that study show that serum retinol was the only carotenoid associated with higher estradiol and testosterone during menses, while beta-carotene, lycopene and lutein were not associated with any reproductive hormones related to the menstrual cycle.

The majority of women who menstruate experience painful and undesirable premenstrual symptoms that last up to 9 days a month. The average age range between menarche and menopause is from 12 to 51 years. This translates into almost 500 cycles in a woman's lifetime. In the present study population, $99 \%$ of women reported experiencing at least one premenstrual symptom (Table 1). While hormone-based medications can significantly decrease symptom discomfort for many women, these medications can be expensive, can be accompanied by various undesirable side effects and have been shown to affect biomarkers of inflammation [32]. Identifying dietary strategies to manage premenstrual symptoms may provide an easily implementable, economical alternative to the cost and potential side effects of hormonal contraceptives. 


\section{Conclusions}

The findings from the present study suggest that higher plasma beta-cryptoxanthin concentrations may be associated with an increase in premenstrual appetite and food cravings. This finding, however, might be due to reverse causation, with an increased premenstrual appetite leading to increased consumption of foods high in beta-cryptoxanthin. No other carotenoids were associated with differences in severity of any premenstrual symptoms after adjusting for multiple comparisons. Further research of the relationship between dietary factors and individual premenstrual symptoms may yield a better understanding of the role of diet in premenstrual symptom prevalence and severity and potentially lead to the development of dietary strategies to manage these symptoms.

Author Contributions: Conceptualization, A.E.-S., S.K., A.J. and B.G.-B.; formal analysis, S.K., T.Z. and A.J.; writing —original draft preparation, S.K., T.Z. and A.J.; writing—review and editing, S.K., T.Z., A.J., B.G.-B. and A.E.-S.; supervision, A.E-S. All authors have read and agreed to the published version of the manuscript.

Funding: This research was funded by the Allen Foundation.

Institutional Review Board Statement: Informed consent was obtained from all participants included in the study, and the study protocol was approved by the Ethics Review Board at the University of Toronto (REB 22587).

Informed Consent Statement: Informed consent was obtained from all subjects involved in the study.

Data Availability Statement: The data presented in this study are available on request from the corresponding author.

Conflicts of Interest: A.E.-S. is the founder of, and holds shares in, Nutrigenomix Inc., a genetic testing company for personalized nutrition. B.G.-B. is the Director of Research and Development at Nutrigenomix Inc. The other authors report no conflicts of interest.

\section{References}

1. Ryu, A.; Kim, T.H. Premenstrual syndrome: A mini review. Maturitas 2015, 82, 436-440. [CrossRef]

2. Leminen, H.; Paavonen, J. PMS and PMDD. Duodecim 2013, 129, 1756-1763.

3. Jarosz, A.C.; Jamnik, J.; El-Sohemy, A. Hormonal contraceptive use and prevalence of premenstrual symptoms in a multiethnic Canadian population. BMC Womens Health 2017, 17, 87. [CrossRef]

4. Adewuya, A.O.; Loto, O.M.; Adewumi, T.A. Pattern and correlates of premenstrual symptomatology amongst Nigerian University students. J. Psychosom. Obstet. Gynecol. 2009, 30, 127-132. [CrossRef]

5. Sternfeld, B.; Swindle, R.; Chawla, A.; Long, S.; Kennedy, S. Severity of Premenstrual Symptoms in a Health Maintenance Organization Population. Obstet. Gynecol. 2002, 99, 1014-1024.

6. Zeitoun, T.; Dehghan Noudeh, N.; Garcia-Bailo, B.; El-Sohemy, A. Genetics of Iron Metabolism and Premenstrual Symptoms: A Mendelian Randomization Study. J. Nutr. 2021, 151, 1747-1754. [CrossRef] [PubMed]

7. Bertone-Johnson, E.R.; Hankinson, S.E.; Willett, W.C.; Johnson, S.R.; Manson, J.E. Adiposity and the development of premenstrual syndrome. J. Womens Health 2010, 19, 1955-1962. [CrossRef] [PubMed]

8. Jarosz, A.C.; El-Sohemy, A. Association between Vitamin D Status and Premenstrual Symptoms. J. Acad. Nutr. Diet. 2019, 119, 115-123. [CrossRef] [PubMed]

9. Jarosz, A.C.; Noori, D.; Zeitoun, T.; Garcia-Bailo, B.; El-Sohemy, A. Variation in the vitamin D receptor gene, plasma 25hydroxyvitamin D, and risk of premenstrual symptoms. Genes Nutr. 2021, 16, 15. [CrossRef] [PubMed]

10. Di Mascio, P.; Murphy, M.E.; Sies, H. Antioxidant defense systems: The role of carotenoids, tocopherols, and thiols. Am. J. Clin. Nutr. 1991, 53, 194s-200s. [CrossRef]

11. Igielska-Kalwat, J.; Gościańska, J.; Nowak, I. Carotenoids as natural antioxidants. Postepy Hig. Med. Dosw. 2015, 69, 418-428. [CrossRef] [PubMed]

12. Bertone-Johnson, E.R.; Ronnenberg, A.G.; Houghton, S.C.; Nobles, C.; Zagarins, S.E.; Takashima-Uebelhoer, B.B.; Faraj, J.L.; Whitcomb, B.W. Association of inflammation markers with menstrual symptom severity and premenstrual syndrome in young women. Hum. Reprod. 2014, 29, 1987-1994. [CrossRef] [PubMed]

13. Sharma, S.; Sheehy, T.; Kolonel, L. Sources of vegetables, fruits and vitamins A, C and E among five ethnic groups: Results from a multiethnic cohort study. Eur. J. Clin. Nutr. 2014, 68, 384-391. [CrossRef]

14. Burrows, T.L.; Williams, R.; Rollo, M.; Wood, L.; Garg, M.L.; Jensen, M.; Collins, C.E. Plasma carotenoid levels as biomarkers of dietary carotenoid consumption: A systematic review of the validation studies. J. Nutr. Intermed. Metab. 2015, 2, 15-64. [CrossRef] 
15. Brossaud, J.; Pallet, V.; Corcuff, J.B. Vitamin A, endocrine tissues and hormones: Interplay and interactions. Endocr. Connect. 2017, 6, R121-R130. [CrossRef]

16. Weber, D.; Grune, T. The contribution of $\beta$-carotene to vitamin A supply of humans. Mol. Nutr. Food Res. 2012, 56, 251-258. [CrossRef]

17. Beltrán-de-Miguel, B.; Estévez-Santiago, R.; Olmedilla-Alonso, B. Assessment of dietary vitamin A intake (retinol, $\alpha$-carotene, $\beta$-carotene, $\beta$-cryptoxanthin) and its sources in the National Survey of Dietary Intake in Spain (2009-2010). Int. J. Food Sci. Nutr. 2015, 66, 706-712. [CrossRef] [PubMed]

18. Al-Delaimy, W.K.; Slimani, N.; Ferrari, P.; Key, T.; Spencer, E.; Johansson, I.; Johansson, G.; Mattisson, I.; Wirfalt, E.; Sieri, S.; et al. Plasma carotenoids as biomarkers of intake of fruits and vegetables: Ecological-level correlations in the European Prospective Investigation into Cancer and Nutrition (EPIC). Eur. J. Clin. Nutr. 2005, 59, 1397-1408. [CrossRef] [PubMed]

19. Chuong, C.J.; Dawson, E.B.; Smith, E.R. Vitamin A levels in premenstrual syndrome. Fertil. Steril. 1990, 54, 643-647. [PubMed]

20. Mira, M.; Stewart, P.M.; Abraham, S.F. Vitamin and trace element status in premenstrual syndrome. Am. J. Clin. Nutr. 1988, 47, 636-641. [CrossRef]

21. Frankel, R.A.; Michels, K.A.; Kim, K.; Kuhr, D.L.; Omosigho, U.R.; Wactawski-Wende, J.; Levine, L.; Perkins, N.J.; Mumford, S.L. Serum antioxidant vitamin concentrations and oxidative stress markers associated with symptoms and severity of premenstrual syndrome: A prospective cohort study. BMC Womens Health 2021, 21, 49. [CrossRef]

22. Bahrami, A.; Bahrami-Taghanaki, H.; Khorasanchi, Z.; Timar, A.; Jaberi, N.; Azaryan, E.; Tayefi, M.; Ferns, G.A.; Sadeghnia, H.R.; Ghayour-Mobarhan, M. Menstrual problems in adolescence: Relationship to serum vitamins A and E, and systemic inflammation. Arch. Gynecol. Obs. 2020, 301, 189-197. [CrossRef] [PubMed]

23. Cahill, L.; Corey, P.N.; El-Sohemy, A. Vitamin C deficiency in a population of young Canadian adults. Am. J. Epidemiol. 2009, 170, 464-471. [CrossRef]

24. Liu, Z.; Lee, H.-J.; Garofalo, F.; Jenkins, D.J.A.; El-Sohemy, A. Simultaneous Measurement of Three Tocopherols, All-trans-retinol, and Eight Carotenoids in Human Plasma by Isocratic Liquid Chromatography. J. Chromatogr. Sci. 2011, 49, 221-227. [CrossRef]

25. Narum, S.R. Beyond Bonferroni. Less conservative analyses for conservation genetics. Cons. Genet. 2006, 7, 783-787. [CrossRef]

26. Burri, B.J.; La Frano, M.R.; Zhu, C. Absorption, metabolism, and functions of $\beta$-cryptoxanthin. Nutr. Rev. 2016, 74, 69-82. [CrossRef]

27. Tang, G. Bioconversion of dietary provitamin A carotenoids to vitamin A in humans. Am. J. Clin. Nutr. 2010, 91, 1468s-1473s. [CrossRef] [PubMed]

28. Puder, J.J.B.C.; Mueller, B.; De Geyter, C.; Dye, L.; Keller, U. Menstrual cycle symptoms are associated with changes in low-grade inflammation. Eur. J. Clin. Investig. 2006, 58-64. [CrossRef]

29. Gold, E.B.; Wells, C.; Rasor, M.O. The Association of Inflammation with Premenstrual Symptoms. J. Womens Health 2016, 25, 865-874. [CrossRef]

30. Dennerstein, L.; Lehert, P.; Heinemann, K. Epidemiology of premenstrual symptoms and disorders. Menopause Int. 2012, 18, 48-51. [CrossRef]

31. Mumford, S.L.; Browne, R.W.; Schliep, K.C.; Schmelzer, J.; Plowden, T.C.; Michels, K.A.; Sjaarda, L.A.; Zarek, S.M.; Perkins, N.J.; Messer, L.C.; et al. Serum Antioxidants Are Associated with Serum Reproductive Hormones and Ovulation among Healthy Women. J. Nutr. 2016, 146, 98-106. [CrossRef] [PubMed]

32. Josse, A.R.; Garcia-Bailo, B.; Fischer, K.; El-Sohemy, A. Novel Effects of Hormonal Contraceptive Use on the Plasma Proteome. PLoS ONE 2012, 7, e45162. [CrossRef] [PubMed] 